\title{
Multistage Graph Problems on a Global Budget
}

\author{
Klaus Heeger \\ TU Berlin, Faculty IV, Algorithmics and Computational Complexity, Germany \\ heeger@tu-berlin.de
}

Anne-Sophie Himmel

TU Berlin, Faculty IV, Algorithmics and Computational Complexity, Germany anne-sophie.himmel@tu-berlin.de

Frank Kammer

THM, University of Applied Sciences Mittelhessen, Giessen, Germany

frank.kammer@mni.thm.de

Rolf Niedermeier

TU Berlin, Faculty IV, Algorithmics and Computational Complexity, Germany rolf.niedermeier@tu-berlin.de

Malte Renken

TU Berlin, Faculty IV, Algorithmics and Computational Complexity, Germany m.renken@tu-berlin.de

\author{
Andrej Sajenko \\ THM, University of Applied Sciences Mittelhessen, Giessen, Germany \\ andrej.sajenko@mni.thm.de
}

\begin{abstract}
Time-evolving or temporal graphs gain more and more popularity when studying the behavior of complex networks. In this context, the multistage view on computational problems is among the most natural frameworks. Roughly speaking, herein one studies the different (time) layers of a temporal graph (effectively meaning that the edge set may change over time, but the vertex set remains unchanged), and one searches for a solution of a given graph problem for each layer. The twist in the multistage setting is that the solutions found must not differ too much between subsequent layers. We relax on this already established notion by introducing a global instead of the local budget view studied so far. More specifically, we allow for few disruptive changes between subsequent layers but request that overall, that is, summing over all layers, the degree of change is moderate. Studying several classical graph problems (both NP-hard and polynomial-time solvable ones) from a parameterized complexity angle, we encounter both fixed-parameter tractability and parameterized hardness results. Somewhat surprisingly, we find that sometimes the global multistage versions of NP-hard problems such as VERTEX COVER turn out to be computationally more tractable than the ones of polynomial-time solvable problems such as MATCHING.
\end{abstract}

2012 ACM Subject Classification Mathematics of computing $\rightarrow$ Graph algorithms; Theory of computation $\rightarrow$ Design and analysis of algorithms

Keywords and phrases Temporal graphs, time-varying graphs, NP-hard problems, Vertex Cover, Matching, parameterized complexity, Cluster Editing

Funding The work started while Frank Kammer and Andrej Sajenko were visiting TU Berlin. Klaus Heeger: Funded by DFG Research Training Group 2434, "Facets of Complexity".

Anne-Sophie Himmel: Funded by DFG, project FPTinP (NI 369/16).

Malte Renken: Funded by the DFG, project MATE (NI 369/17).

Andrej Sajenko: Funded by the DFG - 379157101. 


\section{Introduction}

Recognizing the need to address the continuous evolution of networks and the steady demand for maintenance due to instances changing over time, in 2014 Eisenstat et al. [14 and Gupta et al. [18] introduced what is now known as the "multistage view" on combinatorial optimization problems. Focusing on graphs, roughly speaking the idea is to consider a series of graphs over a fixed vertex set and changing edge set (this is known as the standard model of temporal graphs), and the goal is to find for each graph of the series (called a layer or snapshot of the temporal graph) a solution of the studied computational problem where solutions to subsequent layers are not "too different" from each other 17 For instance, consider the famous VerTex Cover problem. Here, the goal is to find for each layer of the temporal graph a (small) set of vertices covering all edges, guaranteeing that these vertex sets between two subsequent layers differ not too much (the degree of change is upper-bounded by a given parameter) [16]. Thus, this can be interpreted as a conservative (no dramatic changes allowed for the solution sets) view on solving problem instances that evolve over time. Clearly, the static case (no "evolution" takes place, that is, there is only one layer) is a special case, leading to many computational hardness results in this setting based already on the hardness of the static version.

Since the pioneering works of Eisenstat et al. [14] and Gupta et al. [18, who mainly focused on polynomial-time approximation algorithms, there has been quite some further development in studying multistage versions of computational problems. For instance, there have been recent studies on Multistage Matching [3], Multistage Vertex Cover [16], Multistage $s$ - $t$ Path [17, Multistage Knapsack [5], Online Multistage Subset Maximization [4, and Multistage Committee Elections [9]. In particular, due to natural parameterizations in this problem setting such as "number of layers" or "maximum degree of change" between the solutions for subsequent instances, also parameterized complexity studies have recently been started [16].

We modify the meanwhile standard multistage model by moving from a local to a global perspective on the number of allowed changes between solution sets for subsequent instances. Whereas in the original model there is a parameter upper-bounding the maximum degree of change between every pair of subsequent layers of a temporal graph, we now introduce a more global view by only upper-bounding the sum of changes. Intuitively, one may say while we still keep the evolutionary view on dynamically changing instances and corresponding solutions, our new model allows for occasional disruptive changes between subsequent layers while on average the degree of change shall be limited ${ }^{2}$

After providing the formal definitions, we will review our results in Table 1 First, however, we discuss the main findings of our work. We provide results both for classical NP-hard graph problems (including a global multistage version of VERTEx COVER) and classical problems solvable in polynomial time (including a global multistage version of MATCHING). We consider three central parameters: $k$, upper-bounding the size of the solution for a layer; $\ell$, the global budget upper-bounding the total number of changes between all layer solutions; $\tau$, the number of layers (equivalently, the lifetime of the temporal graph). The three key messages of our work are as follows:

1 Without going into any details, we remark that there are similarities between "parameterized multistaging" and parameterized studies of dynamic problems [1, 19, 24, 25, and reoptimization 7 .

2 We are only aware of a Bachelor Thesis [27] supervised by the TU Berlin group where such a global multistage view has been adopted for the specific case of VERTEX COVER. 
Table 1 Overview on our (time) complexity results, where $n$ is the number of vertices of the temporal graph, $k$ is the solution size, $\ell$ is the global budget, and $\tau$ is the lifetime of the temporal graph. Note that the first five problems are NP-hard in the static case, while the last three are polynomial-time solvable. "?" denotes open cases. ${ }^{\dagger}$ indicates that hardness prevails even for $\ell=0$, and therefore also for the classical multistage version. ${ }^{\ddagger}$ indicates that hardness prevails even for planar underlying graphs. Herein, W[1]-h. and W[2]-h. refer to parameterized hardness, poly. kernel refers to the existence of a polynomial-size problem kernel, and para-NP-h. refers to NP-hardness even for constant parameter values.

\begin{tabular}{|c|c|c|c|c|}
\hline Problem & $k$ & $\ell$ & $k+\ell$ & $k+\tau$ \\
\hline VERTEx Cover & $\mathrm{W}[1]-\mathrm{h}$. & para-NP-h. & $k^{O(k+\ell)} \tau \operatorname{poly}(\mathrm{n})$ & poly. kernel \\
\hline Path Contraction & $\mathrm{W}[1]-\mathrm{h}$. & para-NP-h. & $k^{O(k+\ell)} \tau \operatorname{poly}(\mathrm{n})$ & poly. kernel \\
\hline Cluster Editing & $\mathrm{W}[1]-\mathrm{h}$. & para-NP-h. & $1.82^{k(\ell+1)} \tau n^{3}$ & $?$ \\
\hline Cluster Edge Deletion & $\mathrm{W}[1]-\mathrm{h}$. & para-NP-h. & $1.82^{k(\ell+1)} \tau n^{3}$ & $?$ \\
\hline Planar Dominating Set & $\mathrm{W}[2]-\mathrm{h}$. & para-NP-h. & $\mathrm{W}[2]-\mathrm{h}^{\dagger}$ & $?$ \\
\hline Edge Dominating Set & $\mathrm{W}[2]-\mathrm{h}^{\ddagger}$ & para-NP-h. ${ }^{\ddagger}$ & $\mathrm{W}[2]-\mathrm{h} .^{\dagger \ddagger}$ & $?$ \\
\hline$s$ - $t$-РATH & $\mathrm{W}[1]-\mathrm{h}$. & para-NP-h. & $\mathrm{W}[1]-\mathrm{h}^{\dagger}$ & $\mathrm{W}[1]-\mathrm{h}^{\dagger}$ \\
\hline$s-t$-CuT & $\mathrm{W}[2]-\mathrm{h}$. & para-NP-h. & $\mathrm{W}[2]-\mathrm{h}^{\dagger}$ & $?$ \\
\hline MATCHING & $\mathrm{W}[1]-\mathrm{h}$. & para-NP-h. & $\mathrm{W}[1]-\mathrm{h}^{\dagger}$ & $?$ \\
\hline
\end{tabular}

1. We encounter (parameterized) computational hardness results for the single parameters $k$ and $\ell$, and even some combinations of $k, \ell$ and $\tau$; the main technical contribution here is to show W-hardness results for the parameter $k$.

2. There is a systematic algorithmic approach that may lead to fixed-parameter tractability results with respect to the combined parameter $k+\ell$. Indeed, along these lines we also obtain polynomial problem kernels with respect to the combined parameter $k+\tau$. We exemplify this approach by providing corresponding results for global multistage versions of the NP-hard problems Vertex Cover, Cluster Editing, Cluster Edge Deletion, and Path Contraction. Notably, we spot a close link to the concept of full kernels from parameterized enumeration [12]. Moreover, for each of our positive algorithmic results we additionally specify the (bit) space complexity of the corresponding algorithms.

3. Global multistage versions of polynomial-time solvable problems such as Matching, $s$ - $t$-PATH, and $s$ - $t$-CUT turn out to be computationally harder than global multistage versions of the above NP-hard problems. More specifically, we spot W-hardness results for the combined parameter $k+\ell$, and in the case of $s$ - $t$-PATH, even for the combined parameter $k+\tau$. This contrast with corresponding, very recent studies of $s$ - $t$-PATH in the standard multistage model [17].

In summary, our first systematic study of global multistage problems leads to a rich and promising new scenario in the fast growing field of studying temporal graph problems [21, 22.

For classical multistage problems, research mainly focused on approximation algorithms [3, 5, 18. While for Multistage Perfect Matching no polynomial-time $O\left(n^{1-\epsilon}\right)$ approximation is possible unless $\mathrm{P}=\mathrm{NP}$ [3, 18, Multistage Vertex Cover is 2approximable in analogy to its static version [2]. On the parameterized complexity side, Multistage Vertex Cover was shown to be fixed-parameter tractable parameterized by $k+\tau$, while being $\mathrm{W}[1]$-hard parameterized by $k$ even if the symmetric difference of the vertex covers in two successive layers may be at most two [16. Global Multistage VERTEX COVER behaves similarly, being fixed-parameter tractable parameterized by $k+\tau$ and W[1]-hard parameterized by $k$. In addition, we show that Global Multistage Vertex 
Cover is fixed-parameter tractable parameterized by $k+\ell$. Bampis et al. [2] showed that a multistage version of MiN-CUT is still polynomial-time solvable; note that their definition of Multistage Min-Cut differs from ours as we consider a cut to be a set of edges, while they consider a cut to be a set of vertices (these two notions are equivalent except for the number of changes between two solutions).

Our work is structured as follows. In Section 2 we provide basic notation and definitions. In Section 3 we present our general approach for gaining fixed-parameter tractability results for global multistage versions of NP-hard problems. Moreover, we present corresponding parameterized hardness results in Section 4 showing that our fixed-parameter tractability results with combined parameter most likely cannot be improved to the parameterization by the single parameter solution size. In Section 5 we then study global multistage versions of polynomial-time solvable problems and encounter simple but surprising parameterized hardness results, contrasting our more positive results for NP-hard problems in Section 3 We conclude in Section 6

\section{Preliminaries}

For an undirected graph $G=(V, E)$, let $V(G):=V$ and $E(G):=E$. For $v \in V$, let $E(G(v)):=\{\{v, u\} \in E(G)\}$ be the edges incident to $v$ in $G$ and let $N(v)$ be the (open) neighborhood of $v$, i.e., $N(v):=\{u:\{v, u\} \in E(G)\}$. A graph is planar if it can be embedded in the plane, that is, the graph can be drawn in the plane without any crossing edges. For any natural number $x$, let $[x]:=\{1, \ldots x\}$.

- Definition 1 (Temporal Graph). We represent $a$ temporal graph $\mathcal{G}$ using an ordered sequence of static graphs (called layers): $\mathcal{G}=\left\langle G_{1}, G_{2}, \ldots, G_{\tau}\right\rangle$. The subscripts $i \in[\tau]$ indexing the graphs in the sequence are the discrete time steps 1 to $\tau$, where $\tau$ is known as the lifetime of $\mathcal{G}$. The underlying graph $G$ of $\mathcal{G}$ is defined as $V(G):=V\left(G_{1}\right)=\ldots=V\left(G_{\tau}\right)$ and $E(G):=E\left(G_{1}\right) \cup \ldots \cup E\left(G_{\tau}\right)$.

We consider graph problems $\mathcal{X}$ in which we are given a graph $G$ and a nonnegative integer $k$, and we are searching for a set of at most $k$ elements that satisfies some property $\mathcal{P}_{\mathcal{X}}$ in $G$. As an example consider the Vertex Cover problem, in which we search for a set of at most $k$ vertices with the property $\mathcal{P}_{\text {Vertex Cover }}$ that these vertices cover all edges of $G$. Such a problem $\mathcal{X}$ is called monotone if every superset of a solution still satisfies $\mathcal{P}_{\mathcal{X}}$ (Vertex Cover being an example of a monotone problem).

We now generalize such problems from graphs to temporal graphs as follows.

- Definition 2 (Global Multistage Problem for graph property $\mathcal{P}_{\mathcal{X}}$ ). Given a triple $(\mathcal{G}, k, \ell)$ where $\mathcal{G}=\left\langle G_{1}, \ldots, G_{\tau}\right\rangle$ is a temporal graph, the goal is to find sets $S_{1}, \ldots, S_{\tau}$, each of size at most $k$, such that $S_{i}$ satisfies $\mathcal{P}_{\mathcal{X}}$ in $G_{i}(i \in[\tau])$ and the total number of insertions from $S_{i}$ to $S_{i+1}$ over all $i \in[\tau-1]$ is upper-bounded by $\ell$, that is, $\sum_{i \in[\tau-1]}\left|S_{i+1} \backslash S_{i}\right| \leq \ell$.

Note that $k$ upper-bounds the solution size in each step whereas $\ell$ upper-bounds the total number of so-called relocations. For some applications, it might make more sense to bound the number of deletions instead of insertions or the sum of insertions and deletions. However, note that while in Definition 2 we only upper-bound the number of insertions from $S_{i}$ to $S_{i+1}$ explicitly, the number of deletions is implicitly upper-bounded by $\ell+\left|S_{1}\right|-\left|S_{\tau}\right|$. Furthermore, for monotone problems removing elements from the solution set is never beneficial except to make space for other elements. Thus, any given solution of a monotone problem can be modified to satisfy $\left|S_{1}\right|=\left|S_{2}\right|=\cdots=\left|S_{\tau}\right|$, so the numbers of insertions and deletions will be equal. 
Parameterized Algorithmics. A parameterized problem $\mathcal{X}$ is fixed-parameter tractable (FPT) if there exists an algorithm solving any instance $(I, k)$ of $\mathcal{X}$ in $f(k) \cdot|I|^{c}$ time, where $f$ is some computable function and $c$ is some constant. If $\mathcal{X}$ is shown to be $\mathrm{W}[1]-$ or $\mathrm{W}[2]$ hard, then it is presumably not fixed-parameter tractable. To show W[1]- or W[2]-hardness, one employs parameterized reductions, that is, algorithms that map any instance $(I, k)$ in $f(k) \cdot|I|^{O(1)}$ time to an equivalent instance $\left(I^{\prime}, k^{\prime}\right)$ with $k^{\prime}=g(k)$ for some computable functions $f, g$. A reduction to a problem kernel is a polynomial-time algorithm that, given an instance $(I, k)$ of $\mathcal{X}$, returns an equivalent instance $\left(I^{\prime}, k^{\prime}\right)$, such that $\left|I^{\prime}\right|+k^{\prime} \leq g(k)$ for some computable function $g$. We call $\left(I^{\prime}, k^{\prime}\right)$ a kernel of $(I, k)$. Problem kernels are usually achieved by applying data reduction rules. Given an instance $(I, k)$, a data reduction rule computes in polynomial time a new instance $\left(I^{\prime}, k^{\prime}\right)$. We call a data reduction rule safe if $(I, k) \in \mathcal{X} \Longleftrightarrow\left(I^{\prime}, k^{\prime}\right) \in \mathcal{X}$. Clearly, for a decidable problem the existence of a problem kernel implies fixed-parameter tractability.

\section{FPT-Frameworks for NP-hard Problems Gone Globally Multistage}

In this section, we introduce two similar, systematic approaches to show fixed-parameter tractability for the combined parameter solution size $k$ plus number $\ell$ of relocations, that is $k+\ell$. This applies to the global multistage versions of NP-hard problems that either are superset-enumerable (see below) or that admit a certain type of kernel and are monotone. We exemplify our two frameworks by applying them to the global multistage versions of the NP-hard problems Cluster Edge Deletion, Cluster Editing, Vertex Cover, and PATH CONTRACTION.

\subsection{Superset-Enumerable Problems}

Our first framework works for problems with the property that all solutions containing some given set $F$ and being minimal under this condition can be enumerated in FPT time.

- Definition 3. A graph property $\mathcal{P}_{\mathcal{X}}$ is superset-enumerable of size $f$ for some computable function $f$ if, for any graph on $n$ vertices, any integer $k$, and any set $F$, the set

$$
\left\{S \supseteq F \mid S \text { satisfies } \mathcal{P}_{\mathcal{X}} \text { in } G \text { and }|S| \leq k\right\}
$$

has at most $f(k)$ minimal elements, and these can be enumerated in poly $(n) f(k)$ time.

Let $\mathcal{P}_{\mathcal{X}}$ be a graph property that is superset-enumerable. Then our framework solves an instance $\left(\mathcal{G}=\left\langle G_{1}, \ldots, G_{\tau}\right\rangle, k, \ell\right)$ of the Global Multistage Problem for $\mathcal{P} \mathcal{X}$, roughly as follows: Guess a minimal solution in the last layer. Extend this solution backwards layer by layer as follows. If the solution $S_{i}(i=\tau, \ldots, 1)$ is also a solution in the previous layer $G_{i-1}$, then set $S_{i-1}:=S_{i}$. Otherwise, guess a subset $F \subseteq S_{i}$. Then enumerate all minimal solutions containing $F$ in $G_{i-1}$ and guess which of them we should take. The superset-enumerability ensures that we can compute all these minimal solutions fast enough as well as that their number is bounded.

We now describe the algorithm in detail.

Step 1 Guess a sequence $L$ of at most $2 \ell+k$ integer pairs $(x, y)$ where $0 \leq x \leq k$ and $0 \leq y \leq f(k)$ such that for each pair either $x=0$ or $y=0$ holds.

Intuitively, a pair $(x, y) \in L$ with $x \neq 0$ instructs us to delete the $x$ th element of the current solution set $S$, and continue with the next pair. If $x=0$, then we enumerate all solutions containing the current solution set $S$, and pick the $y$ th such solution as the new 
current solution (where the elements of $S$ and the solutions are numbered in an arbitrary order).

Step 2 Start with $S=\emptyset$ and $i=\tau$. Repeat this step as long as $i \geq 1$. If $S$ does not satisfy $\mathcal{P}_{\mathcal{X}}$ on $G_{i}$, then take (and remove) the first pair of $L$ and modify $S$ accordingly as described above. Otherwise, if $S$ satisfies $\mathcal{P}_{\mathcal{X}}$ on $G_{i}$, then set $S_{i}:=S$ and decrement $i$.

Step 3 If at any point we try to take an element from $L$ while it is empty, the sum $\sum_{i \in[\tau-1]}\left|S_{i+1} \backslash S_{i}\right|$ exceeds $\ell$, or the size $|S|$ exceeds $k$, then restart from Step 1. Otherwise, the sets $S_{1}, \ldots, S_{\tau}$ form a solution for $(\mathcal{G}, k, \ell)$ for graph property $\mathcal{P}_{\mathcal{X}}$.

- Theorem 4. Let $\mathcal{P}_{X}$ be a graph property that is superset-enumerable of size $f$. Given an n-vertex temporal graph $\mathcal{G}=\left\langle G_{1}, \ldots, G_{\tau}\right\rangle$ as well as integers $k$ and $\ell$, the GLOBAL Multistage Problem $(\mathcal{G}, k, \ell)$ for $\mathcal{P}_{\mathcal{X}}$ can be solved in poly $(n) \tau(k+f(k)+1)^{2 \ell+k}$ time and using $O((\ell+k) \log f(k)+k \log n+\log \tau)$ bits in addition to the space needed to enumerate and verify the solutions.

Proof. Since each element of $L$ can take $k+f(k)$ possible values, the number of sequences of length at most $2 \ell+k$ is bounded by $(k+f(k))^{2 \ell+k}$. Thus, the running time of our algorithm can be upper-bounded by poly $(n) \tau(k+f(k)+1)^{2 \ell+k}$. Storing $L, S$ and $i$ can be done in the space bound described in the theorem. The entire sequence $S_{1}, \ldots, S_{\tau}$ does not need to be stored at any point. Instead, once a correct guess for $L$ has been found and verified, $L$ can be used to recompute and immediately output $S_{1}, \ldots, S_{\tau}$ one after another.

We next show that the algorithm solves the Global Multistage Problem. Assume that the algorithm returns a solution $\left(S_{1}, \ldots, S_{\tau}\right)$. Note that our checks in Step 3 guarantee that $\left(S_{1}, \ldots, S_{\tau}\right)$ is a valid solution.

Now assume that there exists a solution $\left(S_{1}, \ldots, S_{\tau}\right)$ for $\left(G_{1}, \ldots, G_{\tau}\right)$. First, note that we may assume that $S_{\tau}$ is a minimal solution for $G_{\tau}$, as we only count insertions. This means that our algorithm finds $S_{\tau}$. Second, note that if $S_{i}$ fulfills $\mathcal{X}$ in $G_{i-1}$ for some $i \in\{2, \ldots, \tau\}$, then $\left(S_{1}, \ldots, S_{i-2}, S_{i}, S_{i}, S_{i+1}, \ldots, S_{\tau}\right)$ is also a solution since replacing $S_{i-1}$ by $S_{i}$ increases the total number of insertions by $\left|S_{i} \backslash S_{i-2}\right|-\left(\left|S_{i} \backslash S_{i-1}\right|+\left|S_{i-1} \backslash S_{i-2}\right|\right) \leq 0$. Intuitively speaking, we can change elements in the solution as late as possible while going backwards through the graphs in $\left(G_{1}, \ldots, G_{\tau}\right)$.

Third note that if an $S^{*}$ fulfills $\mathcal{P}_{\mathcal{X}}$ in $G_{i-1}$ with $S_{i-1} \cap S_{i} \subseteq S^{*} \subseteq S_{i-1}$, then $\left(S_{1}, \ldots, S_{i-2}, S^{*}, S_{i}, \ldots S_{\tau}\right)$ is also a solution, as $\left|S_{i} \backslash S^{*}\right|+\left|S^{*} \backslash S_{i-2}\right| \leq\left|S_{i} \backslash S_{i-1}\right|+\left|S_{i-1} \backslash S_{i-2}\right|$ follows from the fact that $\left(S_{i} \backslash S^{*}\right) \cup\left(S^{*} \backslash S_{i-2}\right) \subseteq\left(S_{i} \backslash S_{i-1}\right) \cup\left(S_{i-1} \backslash S_{i-2}\right)$. This third point actually generalizes the second observation.

To sum up, we may assume that there is a solution $\left(S_{1}, \ldots, S_{\tau}\right)$ such that

- $S_{\tau}$ is a minimal solution,

- if $S_{i}$ fulfills $\mathcal{P}_{\mathcal{X}}$ in $G_{i-1}$, then $S_{i-1}=S_{i}$, and

- $S_{i-1}$ is minimal amongst all solutions of size at most $k$ containing $S_{i} \cap S_{i-1}$.

Since our algorithm ultimately explores all sequences of this form, we may conclude that it will eventually find a solution if it exists.

We now show that our framework is applicable to all monotone problems admitting a full kernel [12, i.e., a kernel containing every minimal solution.

- Proposition 5. Let $\mathcal{X}$ be a monotone problem admitting a full kernel of size $f^{\prime}(k)$, where $k$ is the solution size bound. Assume that the graph property $\mathcal{P}_{\mathcal{X}}$ can be verified in polynomial time. Then $\mathcal{P}_{\mathcal{X}}$ is superset-enumerable of size $f(k)=2^{f^{\prime}(k)}$. 
Proof. Given a graph $G$ and any set $F$, we compute a full kernel $K$ of $G$. For each subset $X \subseteq K \backslash F$, we check whether $X \cup F$ satisfies $\mathcal{P}_{\mathcal{X}}$, has size at most $k$, and whether $X$ is minimal with these properties (since $\mathcal{X}$ is monotone, checking for minimality can be done by checking for each element $x \in X$ whether $(F \cup X) \backslash\{x\}$ satisfies $\left.\mathcal{P}_{\mathcal{X}}\right)$. This clearly runs in FPT-time and returns at most $2^{f^{\prime}(k)}$ solutions since $f^{\prime}(k)$ is an upper bound for the size of the full kernel $K$. Also any solution returned is minimal under all solutions containing $F$, so it remains to show that every solution $S$ with this property is returned. To see this, let $S^{\prime} \subseteq S$ be a minimal solution (not necessarily containing $F$ ). We have $S^{\prime} \backslash F \subseteq S^{\prime} \subseteq K$ since $K$ is a full kernel. As $\mathcal{X}$ is monotone, $S^{\prime} \cup F \subseteq S$ is also a solution, thus $S^{\prime} \cup F=S$ by minimality of $S$. Thus, when the algorithm tests $X=S^{\prime} \backslash F \subseteq K \backslash F$, it will output the solution $S$.

Combining Theorem 4 and Proposition 5 we obtain the following.

- Corollary 6. Let $\mathcal{X}$ be a monotone graph problem having a kernel of size $f^{\prime}(k)$ for the solution size bound $k$ and some computable function $f^{\prime}$. Given an n-vertex temporal graph $\mathcal{G}=\left\langle G_{1}, \ldots, G_{\tau}\right\rangle$ as well as parameters $k$ and $\ell$, the Global Multistage Problem $(\mathcal{G}, k, \ell)$ for graph property $\mathcal{P}_{\mathcal{X}}$ can be solved in $\operatorname{poly}(n) \tau\left(\max \left\{k+1,2^{f^{\prime}(k)}+1\right\}\right)^{2 \ell+k}$ time and $O\left((\ell+k) f^{\prime}(k)+k \log n+\log \tau\right)$ bits in addition to the space needed to compute the full kernels and to verify the solutions.

\subsection{Monotone Problems with Full Kernels}

For our second framework, which only targets monotone problems with a full kernel, we now slightly modify our approach from the last section to improve the parts of the time bounds that depend on the parameters $k$ and $\ell$. This allows us to get improved bounds for the global multistage versions of the NP-hard problems Vertex Cover and Path Contraction. In addition, our second framework allows us to compute kernels for these two problems.

Let $\mathcal{X}$ be a parameterized monotone problem that admits a full kernel and that allows for any solution to be verified in time polynomial in the input instance size. Then, for an instance $\left(\mathcal{G}=\left\langle G_{1}, \ldots, G_{\tau}\right\rangle, k, \ell\right)$ of the Global Multistage Problem for graph property $\mathcal{P}_{\mathcal{X}}$, the rough idea behind our framework is as follows: For each layer $G_{i}$ of the temporal graph, we compute a kernel $G_{i}^{\prime}$. Now we can guess the insertions and replacements from solution $S_{1}$ to $S_{\tau}$. This approach exploits two properties of $\mathcal{X}$ to make this approach work. Due to monotonicity, we never have to delete any element except if we aim to replace it, and the full kernel ensures that each layer contains all minimal solutions of size $k$ for that layer. In the following, we call the reductions that are used to get these instances full reductions. We remark that, while a full kernel is required to contain all elements of each minimal solution of size at most $k$, our framework can easily be adapted to only require that each element of each minimal solution of size at most $k$ is uniquely represented by some arbitrary element of the kernel.

We next describe our framework for solving the Global Multistage Problem $(\mathcal{G}, k, \ell)$ for graph property $\mathcal{P}_{\mathcal{X}}$ on a temporal graph $\mathcal{G}=\left\langle G_{1}, \ldots, G_{\tau}\right\rangle$ with lifetime $\tau$. Even though we can apply the framework of the previous section to full kernels of size $f(k)$ by enumerating all possible solutions in the kernel, at every step we have to essentially guess a number in $1, \ldots,\left(2^{f(k)}+1\right)$. In contrast, the revised framework below only requires guesses from $1, \ldots,(f(k)+1)$.

To simplify the algorithm and the following proof, we will replace the requirement $\sum_{i \in[\tau-1]}\left|S_{i+1} \backslash S_{i}\right| \leq \ell(*)$ by the modified bound $\left|S_{1}\right|+\sum_{i \in[\tau-1]}\left|S_{i+1} \backslash S_{i}\right| \leq k+\ell(* *)$ which is clearly implied by $(*)$. We argue that this does not constitute a restriction because 
for any solution that obeys $(* *)$, the algorithm can do the following. As long as $(*)$ does not hold, take $i$ as the first time step where $N:=S_{i+1} \backslash S_{i}$ is not empty, pick an arbitrary element from $N$ and add it to all of $S_{1}, \ldots, S_{i}$. It is easy to check that this will yield a solution for $(*)$.

We now give the algorithm for solving the Global Multistage Problem.

Step 0 Compute a full kernel $G_{i}^{\prime}$ for all $G_{i}(i \in[\tau])$. In this way, we obtain a sequence of kernels $\mathcal{G}^{\prime}=\left\langle G_{1}^{\prime}, \ldots, G_{\tau}^{\prime}\right\rangle$.

Step 1 Guess a sequence $L$ of at most $\ell+k$ integer pairs $(x, y)$ where $1 \leq y \leq f(k)$ as well as $x=0$ for the first $k$ integer pairs and $1 \leq x \leq f(k)$ for the remaining. Intuitively, a pair $(x, y) \in L$ with $x \neq 0$ instructs us to replace the $x$ th element of the current solution set $S$ with the $y$ th element of $G_{i}^{\prime}$. If $x=0$, then only the $y$ th vertex is added.

Step 2 Start with $S=\emptyset$ and $i=1$. Repeat this step as long as $i \leq \tau$. If $S$ does not satisfy $\mathcal{P}_{\mathcal{X}}$ on $G_{i}^{\prime}$, then take (and remove) the first pair of $L$ and modify $S$ accordingly as described above. Otherwise, if $S$ satisfies $\mathcal{P}_{\mathcal{X}}$ on $G_{i}^{\prime}$, then set $S_{i}:=S$ and increment $i$.

Step 3 If at any point we try to take an element from $L$ while it is empty, or if $|S|>k$, then restart from Step 1. Otherwise, the sets $S_{1}, \ldots, S_{\tau}$ form a solution for $(\mathcal{G}, k, \ell)$ for graph property $\mathcal{P}_{\mathcal{X}}$.

- Theorem 7. Let $\mathcal{X}$ be a monotone parameterized graph problem such that it admits a full kernel of size $f(k)$ and any solution can be verified in polynomial time. Given an n-vertex temporal graph $\mathcal{G}=\left\langle G_{1}, \ldots, G_{\tau}\right\rangle$ as well as parameters $k$ and $\ell$, the following holds for the Global Multistage Problem $(\mathcal{G}, k, \ell)$ for graph property $\mathcal{P}_{\mathcal{X}}$ :

1. it can be solved in $f(k)^{2 \ell+k} \tau \operatorname{poly}(n)$ time and $O((\ell+k) \log f(k)+\log \tau)$ bits in addition to the space needed to compute the full kernels and to verify the solutions.

2. if there is a polynomial time computable function $\phi$ which maps a graph $G=(V, E)$ and a vertex set $W$ to a graph $\phi(G, W)$ with $V(\phi(G, W))=V \cup W$ such that $S \subseteq V \cup W$ satisfies $\mathcal{P}_{\mathcal{X}}$ in $\phi(G, W)$ if and only if $S \cap V$ satisfies $\mathcal{P}_{\mathcal{X}}$ in $G$, then the Global Multistage Problem has a kernel of at most $f(k) \tau$ vertices and at most $(f(k) \tau)^{2} \tau$ edges.

Proof. The running time of our algorithm can be upper-bounded by $f(k)^{2 \ell+k} \tau \cdot \operatorname{poly}(n)$ as there are $2 \ell+k$ numbers to be guessed from $1, \ldots, f(k)$ and the algorithm takes $\tau \cdot \operatorname{poly}(n)$ time for each attempt. The space bound in the theorem allows us to store $L, S$ and $i$.

It remains to show that the algorithm finds a solution with bound (**) for $\mathcal{G}$ if it exists. Without loss of generality, we can assume that the solution for $\mathcal{G}$ changes the vertices/edges over time as late as possible. In particular we first can do all insertions and then all replacements. By our guess, we determine the vertices of the solution $S_{1}$ in $G_{1}$. Furthermore, whenever the vertices in the solution set change, we also can guess that change in our sequence of pairs. Knowing $S_{1}$ and the sequence, our algorithm finds the solution for $\mathcal{G}$. Finally note that if some instance $\left(G_{i}, k\right)$ is a no-instance, then our algorithm finds also no solution.

We now show how to turn the sequence of full kernels $\left(G_{i}^{\prime}\right)_{i \in[\tau]}$ into a kernel for the global multistage problem. Note that the latter must have the same same vertex set in every time step.

Let $W:=\bigcup_{i \in[\tau]} V\left(G_{i}^{\prime}\right)$ and note that $|W| \leq f(k) \tau$. Then $\left\langle\phi\left(G_{i}^{\prime}, W\right)\right\rangle_{i \in[\tau]}$ is a temporal graph whose number of edges is clearly at most $(f(k) \tau)^{2} \tau$. Due to the conditions placed on the function $\phi,\left(\left\langle\phi\left(G_{i}^{\prime}, W\right)\right\rangle_{i \in[\tau]}, k, \ell\right)$ is a kernel for the Global Multistage Problem for $\mathcal{P}_{\mathcal{X}}$. 
The running time of our algorithm can be easily improved if the solution set of a monotone graph problem $\mathcal{X}$ only consists of vertices - as it is often the case. Then we can replace the $f(k)$-term in the running time by the number of vertices in the kernel.

\subsection{Applications}

Cluster Editing and Cluster Edge Deletion. We start to show that our framework for superset-enumerable problems can be applied very easily to the following two clustering problems.

The first problem is Cluster Editing (also known as Correlation Clustering) where the goal is to find a modification of a given graph by deleting or adding edges such that the modified graph is a cluster graph, i.e., all connected components of the modified graph are cliques (also called clusters).

$\triangleright$ Problem (Global Multistage Cluster Editing). Given a triple $(\mathcal{G}, k, \ell)$ where $\mathcal{G}=\left\langle G_{1}, \ldots, G_{\tau}\right\rangle$ is a temporal graph, the goal is to find sets $S_{1}, \ldots, S_{\tau} \subseteq E(\mathcal{G}) \times\{$ del $\} \cup$ $\{\{u, v\}: u, v \in V(\mathcal{G})\} \times\{$ add $\}$, each of size at most $k$, such that $\left(G_{i} \backslash\left\{e \in E\left(G_{i}\right)\right.\right.$ : $(e$, del $\left.\left.) \in S_{i}\right\}\right) \cup\left\{e:(e\right.$,add $\left.) \in S_{i}\right\}$ is a cluster graph for $i \in[\tau]$ and the total number of new elements when going from $S_{i}$ to $S_{i+1}(i \in[\tau-1])$ is upper-bounded by $\ell$, that is, $\sum_{i \in[\tau-1]}\left|S_{i+1} \backslash S_{i}\right| \leq \ell$.

We mention in passing that Cluster EDITING in temporal graphs has also been studied by Chen et al. [11] and a related dynamic model by Luo et al. [25].

The second problem is Cluster Edge Deletion. It is the restriction of Cluster Editing where one is only allowed to delete edges. Global Multistage Cluster Edge Deletion is the restriction of Global Multistage Cluster Editing with $S_{i} \subseteq E(\mathcal{G}) \times$ $\{$ del $\}$ for all $i \in[\tau]$.

- Corollary 8. For an n-vertex, $\tau$-layer temporal graph $\mathcal{G}$, the Global Multistage Cluster Editing and Global Multistage Cluster Edge Deletion instances $(\mathcal{G}, k, l)$ can both be solved in $1.82^{k(\ell+1)} \tau n^{3}$ time. An extra factor of $(\log k)$ in the running time allows us to solve both problems with $O((\ell+k) k \ell+k \log n+\log \tau)$ bits.

Proof. The claim follows directly from Theorem 4 and the fact that both problems are $\left(1.82^{k}, n^{3}\right)$-enumerable, which can be done by making the modification of an edge from the subset $S$ more expensive than the budget $k$, and then applying Böcker et al.'s search-tree algorithm for Weighted Cluster Editing [8, Theorem 7] (note that this algorithm can also be used for Cluster EdGe Deletion by setting the weight of adding an edge to $k+1$ ).

The algorithm runs in $O\left(1.82^{k}+n^{3}\right)$ time, modifies the graph and builds a search tree of depth $O(k)$. Each descending step of the search tree makes $O(1)$ changes to the graph. These changes as well as our modifications due to subset $S$ can be maintaines in a heap with $O(k \log n)$ bits. Evaluating the given graph and the heap, an access to the modified graph runs in $O(\log k)$ time. By Theorem 4 the space bound in the corollary follows.

Vertex Cover. As a first simple application of our framework for full kernels, we consider Global Multistage Vertex Cover. In a graph $H, C \subseteq V(H)$ is a vertex cover if for every $\{u, v\} \in E(H): v \in C$ or $u \in C$. In the NP-hard Vertex Cover problem, we are given $(H, k)$ where $H$ is a graph and $k \in \mathbb{N}$. The goal is to find a vertex cover of size at most $k$ in $H$. It is worth noting that VERTEX COVER has already been investigated in the classical multistage scenario by Fluschnik et al. [16]. 
$\triangleright$ Problem (Global Multistage Vertex Cover). Given a triple $(\mathcal{G}, k, \ell)$ where $\mathcal{G}=$ $\left\langle G_{1}, \ldots, G_{\tau}\right\rangle$ is a temporal graph, the goal is to find sets $S_{1}, \ldots, S_{\tau}$, each of size at most $k$, such that $S_{i} \subseteq V\left(G_{i}\right)$ is a vertex cover for $G_{i}(i \in[\tau])$ and the total number of insertions from $S_{i}$ to $S_{i+1}(i \in[\tau-1])$ is upper-bounded by $\ell$, that is, $\sum_{i \in[\tau-1]}\left|S_{i+1} \backslash S_{i}\right| \leq \ell$.

For a static $n$-vertex graph $H$, VERTEX COVER with parameter $k$ admits a full kernel of at most $k^{2}+2 k$ vertices and at most $k^{2}+k$ edges by applying the following three data reduction rules (known as Buss kernelization [10]) exhaustively [12]. Moreover, Fafianie and Kratsch [15] have shown that the kernel can be computed within $O(f(k) \log n)$ bits. It is not hard to see that a solution can be verified with $O(\log n)$ bits.

Rule 1. Delete all isolated vertices.

Rule 2. If a vertex $v$ has more than $k+1$ incident edges, then delete all except $k+1$ of them.

Rule 3. If Rules 1 and 2 cannot be applied any more and $H$ has more than $k^{2}+2 k$ vertices or more than $k^{2}+k$ edges, then conclude that the given graph is a no-instance.

Clearly, Rule 1 is a full reduction rule since an isolated vertex can never be part of any minimal solution for $H$. Rule 2 is also full since a vertex $v$ with $k+1$ edges is part of every solution of size $k$, i.e., all deleted edges are covered by $v$. If we apply Rule 3 , then one can easily see that we transform a no-instance to a no-instance. Thus, we get a sequence of full kernels and we can apply our framework as described in Subsection 3.2

To obtain a kernel for an instance $\left(\mathcal{G}=\left\langle G_{1}, \ldots, G_{\tau}\right\rangle, k, \ell\right)$ of Global Multistage Vertex Cover, we can simply apply Theorem 7 (ii) with the function $\phi((V, E), W):=$ $(V \cup W, E)$ since adding isolated vertices does not affect an instance of VERTEX Cover. Note that the resulting kernel contains at most $\left(k^{2}+2 k\right) \tau$ vertices and at most $\left(k^{2}+k\right) \tau$ edges. By Theorem 7, we can conclude the following.

- Corollary 9. For an n-vertex, $\tau$-layer temporal graph $\mathcal{G}$, Global Multistage Vertex COVer on an instance $(\mathcal{G}, k, \ell)$ can be solved in $\left(k^{2}+2 k+1\right)^{2 \ell+k} \tau \cdot \operatorname{poly}(n)$ time and $O((\ell+k) \log f(k)+\log \tau+f(k) \log n)=O\left(f^{\prime}(k, \ell) \log n+\log \tau\right)$ bits for some computable function $f^{\prime}$. Moreover, the problem has a kernel of at most $\left(k^{2}+2 k\right) \tau$ vertices and at most $\left(k^{2}+k\right) \tau$ edges computable in $O(\tau(n+m))$ time, where $m$ is the number of edges in the underlying graph.

Path Contraction. As a second application of our framework for full kernels, we consider the NP-hard problem Path Contraction [20]. For a graph $H$ and a subset of its edges $C \subseteq E(H)$, we write $H / C$ for the graph obtained from $H$ by contracting each edge in $C$. (Contracting an edge means merging its endpoints into a single vertex and removing any loops or parallel edges afterwards.) In the PATH Contraction problem, we are given a graph $H$ and an integer $k \in \mathbb{N}$, and the goal is to find $C \subseteq E(H)$ with $|C| \leq k$ such that every connected component of $H / C$ is a path. Again, the multistage adaption is straightforward.

$\triangleright$ Problem (Global Multistage Path Contraction). Given a triple $(\mathcal{G}, k, \ell)$ where $\mathcal{G}=\left\langle G_{1}, \ldots, G_{\tau}\right\rangle$ is a temporal graph, the goal is to decide whether there exist $S_{1}, \ldots, S_{\tau}$ with $S_{i} \subseteq E(G)$ ( $G$ being the underlying graph of $\mathcal{G}$ ), each of size at most $k$, such that $G_{i} /\left(S_{i} \cap E\left(G_{i}\right)\right)$ is a disjoint union of paths for every $i \in[\tau]$, and the total number of insertions from $S_{i}$ to $S_{i+1}$ is upper-bounded by $\ell$, that is, $\sum_{i \in[\tau-1]}\left|S_{i+1} \backslash S_{i}\right| \leq \ell$.

Path Contraction has a problem kernel with at most $5 k+3$ vertices and at most $(5 k+$ $3)^{2}$ edges with respect to solution size $k[20$, by applying the data reduction rules below on each instance $(H, k)$ of Path Contraction. The kernel can be computed within $O(f(k) \log n)$ bits 23 . We show that this kernel is also a full kernel. 
Rule 1. If any connected component of $H$ contains an edge $e=\{u, v\} \in E(H)$ whose removal disconnects it into two connected components that contain at least $k+2$ vertices each, then contract the edge $e$.

Rule 2. If Rule 1 is not applicable and any connected component has more than $5 k+3$ vertices, then conclude that $(H, k)$ is a no-instance.

Rule 1 is a "full reduction rule" since every path obtained from $H$ after the contraction of $k$ edges has some vertices connected to $u$ and some connected to $v$ so that it does not make sense to contract edge $e$. In other words, every minimal solution of size at most $k$ must not contain edge $e$. Rule 2 is surely full.

To obtain a kernel for an instance of Global Multistage Path Contraction, observe as before that adding isolated vertices does not affect the solution. Hence, the kernel is constructed in the same manner as shown for Global Multistage Vertex Cover. Thus, with Theorem 7 we get the following.

- Corollary 10. For an n-vertex, $\tau$-layer temporal graph $\mathcal{G}$, Global Multistage Path CONTRACTION on an instance $(\mathcal{G}, k, \ell)$ can be solved in $(5 k+4)^{4 \ell+2 k} \tau \cdot \operatorname{poly}(n)$ time and using $O\left(f^{\prime}(k, \ell) \log n+\log \tau\right)$ bits for some computable function $f^{\prime}$. Moreover, the problem has a kernel of at most $(5 k+3) \tau$ vertices and at most $(5 k+3)^{2} \tau$ edges computable in $O(\tau n(n+m))$ time, where $m$ is the number of edges in the underlying graph.

\section{Parameterized Hardness for NP-hard Problems Gone Globally Multistage}

In this section, we explore the limitations with respect to achieving fixed-parameter tractability results of the global multistage versions of NP-hard problems. It is clear that the global multistage version of an NP-hard problem is NP-hard for $\tau=1$ and $\ell=0$ relocations. We further show that the global multistage versions of Vertex Cover, PATH Contraction, and Cluster Edge Deletion are W[1]-hard with respect to solution size $k$. For the global multistage versions of Planar Dominating Set and Planar Edge Dominating Set, we even show W[2]-hardness with respect to the combined parameter solution size $k$ plus number $\ell$ of relocations.

\subsection{Hardness for the Parameter $k$}

In this subsection, we show that the global multistage versions of VERTEx COVER, PATH Contraction and Cluster Edge Deletion become W[1]-hard with respect to solution size $k$. This is each time done by a reduction from Clique, which is well-known to be $\mathrm{W}$ [1]-complete with respect to solution size [13. In CLIQUE, given a graph $H$ and a number $\tilde{k}$, the question is whether $H$ contains $\tilde{k}$ pairwise adjacent vertices. We prove the following.

- Theorem 11. Global Multistage Vertex Cover, Global Multistage Path Contraction, and Global Multistage Cluster Edge Deletion are W[1]-hard parameterized by the solution size $k$.

For the proof of Theorem 11, we describe our parameterized reduction from CLIQUE using Global Multistage Vertex Cover in full detail as an example. We highlight the necessary properties of the corresponding vertex gadget and the edge gadget and prove the correctness of the reduction based on these properties. Afterwards, we show how to build the vertex gadgets and the edge gadgets for PATH CONTRACTION and CLUSTER EdGE DELETION based on similar ideas. 
Before going into details of the reductions, we remark that Theorem 11 also implies the $\mathrm{W}$ [1]-hardness of a variant of the global multistage scenario, in which the solution for two successive time layers may differ only by $q \geq 1$ elements. A formal definition of this variant is as follows.

- Definition 12 (Global Multistage with $q$-Local Budget for graph property $\mathcal{P}_{X}$ ). Given an instance $(\mathcal{G}, k, \ell)$ of Global Multistage for graph property $\mathcal{P}_{X}$, the goal is to find a solution $\mathcal{S}=\left(S_{1}, \ldots, S_{\tau}\right)$ to the Global Multistage problem $(\mathcal{G}, k, \ell)$ with the additional restriction that $\left|S_{i+1} \backslash S_{i}\right| \leq q$ for all $i \in[\tau-1]$.

We then obtain the following corollary to Theorem 11.

- Corollary 13. Global Multistage Vertex Cover with local Budget, Global Multistage Path Contraction with local Budget, and Global Multistage Edge Deletion with $q$-Local Budget parameterized by $k$ are $\mathrm{W}[1]$-hard for any fixed $q \geq 1$.

Proof. Given an instance Global Multistage Vertex Cover (Path Contraction, Cluster Edge Deletion), add $k$ empty layers, i.e., $k$ layers with no edges between any two successive layers of the input temporal graph.

Since any set of vertices is a solution to the added empty layers, all changes to the solution set can be performed element by element in the empty layers. Thus, the instances are equivalent.

\subsubsection{Vertex Cover}

Let $(H, \tilde{k})$ be an instance of Clique. Let $V(H)=\left\{v_{1}, v_{2}, \ldots, v_{n}\right\}$ and $E(H)=\left\{e_{1}, e_{2}, \ldots, e_{m}\right\}$. We construct a temporal graph $\mathcal{G}$ as follows.

Vertex gadget. For each vertex $v_{i} \in V(H)$, the graph $\mathcal{G}$ contains a set $V_{i}$ of $4 \tilde{k}$ vertices $v_{i}^{1}, \ldots, v_{i}^{4 \tilde{k}}$. We call this set $V_{i}$ the copy set of $v_{i}$.

Edge gadget. An edge gadget $\mathcal{G}_{e}$ for an edge $e=\left\{v_{p}, v_{q}\right\}$ in $H$ adds $8 \tilde{k}^{2} n+1$ layers and the vertices $w_{e}^{1}, w_{e}^{2}, \ldots, w_{e}^{8 \tilde{k}^{2} n}, w_{e}^{*}$ to the temporal graph. For $i \in[n]$ and $j \in\left[8 \tilde{k}^{2}\right]$, in the $(i+j n)$-th layer of $G_{e}$, vertex $w_{e}^{i+j n}$ is the only non-isolated vertex not contained in a copy set. It is connected to all vertices in $V_{i}$. In the $\left(8 \tilde{k}^{2} n+1\right)$-st layer, vertex $w_{e}^{*}$ is the only non-isolated vertex not contained in a copy set. It is connected to all vertices in $V_{q}$ and $V_{p}$. Let $W$ be the set of vertices consisting of all $w_{e}^{i}$ and all $w_{e}^{*}$ over all edges $e \in E(H)$ and $i \in\left[8 \tilde{k}^{2} n\right]$. See Figure 1 for an example.

Instance. In the first time layer, we have a clique consisting of $4 \tilde{k}^{2}+2$ new vertices $V^{\text {first }}$. The vertices of this clique are isolated in all other layers. The temporal graph contains $m$ edge gadgets, one for each edge of $H$. We set $k:=4 \tilde{k}^{2}+1$ and $\ell=4 \tilde{k}^{2}+8 m \tilde{k}^{2}(n-\tilde{k})+\left(m-\left(\begin{array}{c}\tilde{k} \\ 2\end{array}\right)\right)$. Note that all layers but the first contain one star with a center being a vertex $w \in W$ called center vertex.

Before we discuss properties of a solution of our Global Multistage Vertex Cover instance, we want to make some basic observation concerning the solution in each layer. First, for any $i \in[n]$ and $j \in\left[8 \tilde{k}^{2}\right]$, in the $(i+j n)$-th layer of an edge gadget for an edge $e$, the whole copy set $V_{i}$ is contained in $S_{i+j n}$, or $w_{e}^{i+j n}$ is contained in $S_{i+j n}$. Vice versa, both $V_{i}$ and $w_{e^{i+j n}}$ form a vertex cover in this layer. Each edge gadget contains $8 \tilde{k}^{2}$ of such layers for each vertex $v_{i} \in V(H)$. 


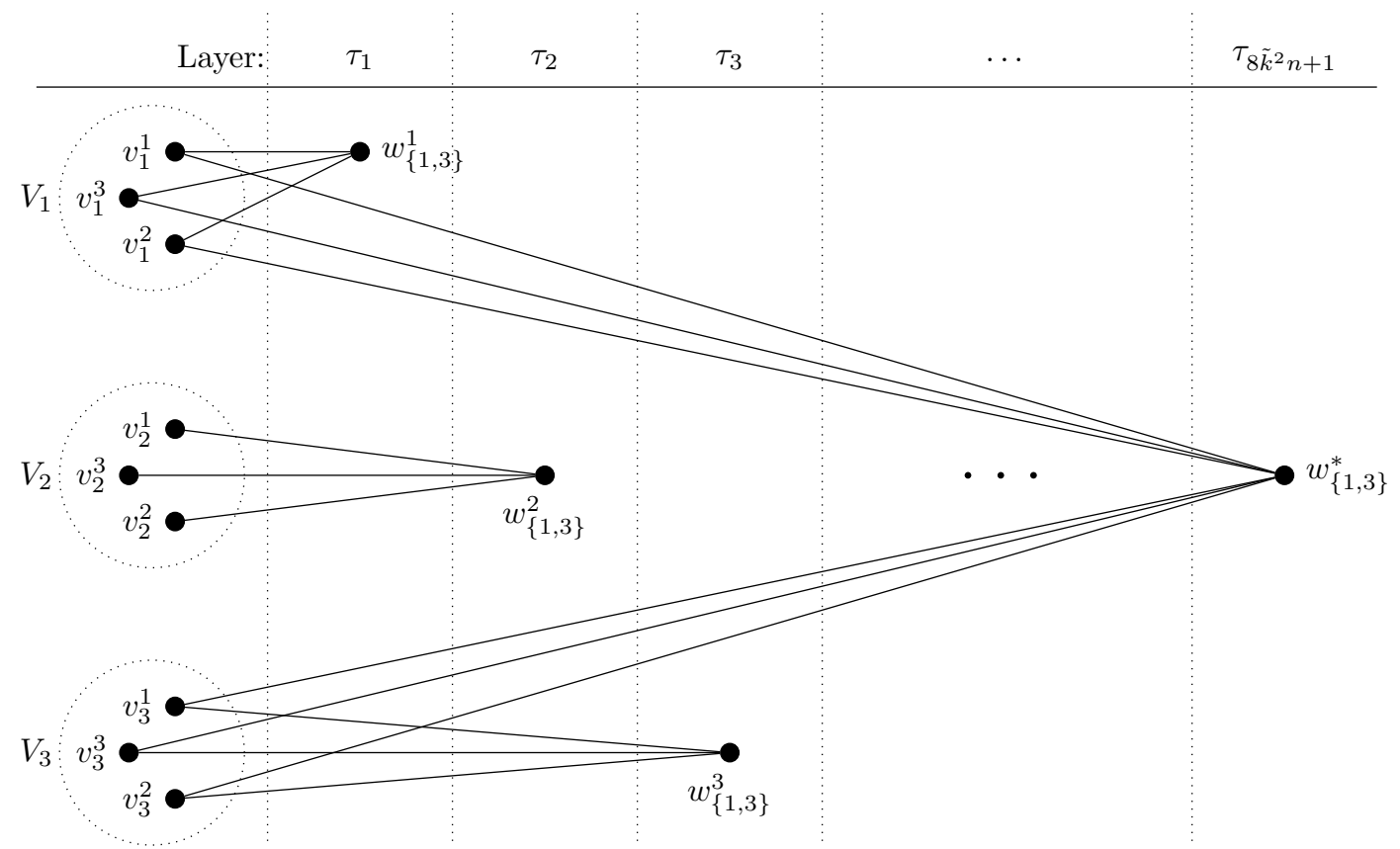

Figure 1 An example of the edge gadget for the edge $e=\left\{v_{1}, v_{3}\right\}$ in a graph with three vertices $v_{1}, v_{2}$, and $v_{3}$ for $\tilde{k}=3$. The $8 \tilde{k}^{2}$ repetitions of layers 1 to 3 (with different center vertices!) are not drawn to keep the figure readable. Furthermore, each copy set contains only three instead of twelve vertices. The edges are only contained in the layer in which the right end vertex $\left(w_{e}^{i}\right.$ or $\left.w_{e}^{*}\right)$ lies. The copy sets are encircled by dotted lines.

Also note that in the last layer of an edge gadget for an edge $e=\left\{v_{p}, v_{q}\right\}$, the copy sets $V_{p}$ and $V_{q}$ are contained in $S_{8 \tilde{k}^{2} n+1}$, or $w_{e}^{*}$ is contained in $S_{8 \tilde{k}^{2} n+1}$. Vice versa, both $V_{p} \cup V_{q}$ and $w_{e}^{*}$ are a vertex cover in this layer.

Properties of the reduction. We start to prove that every solvable instance has a solution $S_{1}, \ldots, S_{\tau}$ with the following properties. Note that the modifications done to prove one property do not destroy the each time earlier properties.

1. $S_{1}$ consists of all but one of the $k+1$ vertices inducing the clique in the first layer.

Proof. To cover the edges of the clique of size $4 \tilde{k}^{2}+2=k+1$ in the first layer, we need $k$ vertices, i.e., the maximum number of allowed vertices.

2. For each layer $i,\left|S_{i} \cap W\right| \leq 1$.

Proof. By Property 1, we can postpone adding $w \in W$ into the solution set until $w$ is not isolated in some layer $i$. Since $w$ is isolated in all subsequent layers $j>i$, we can replace $w$ whenever another vertex $w^{\prime} \in W$ is not isolated and should be added to the solution set.

3. $S_{2}$ contains $\tilde{k}$ copy sets.

Proof. The vertices in $S_{1}$ are isolated in all layers but the first one. Thus we may assume that these vertices are relocated before any vertices from $\bigcup_{i} V_{i}$. Furthermore we may assume that all these relocations already happen in the second layer as postponing them does not gain anything. Note that relocating $\left|V_{i}\right|$ vertices from $S_{1}$ to some copy set $V_{i}$ initially costs $4 \tilde{k}$ relocations, but overall saves $8 \tilde{k}^{2}$ relocations inside $W$ in the first edge gadget. Thus, each copy set entirely contained in $S_{2}$ decreases the overall cost of the first 
edge gadget. Since $k=\tilde{k}\left|V_{i}\right|+1$, this means that $S_{2}$ contains exactly $\tilde{k}$ copy sets entirely.

4. For each layer $i$ and each copy set $V_{j}$ : either $\left|S_{i} \cap V_{j}\right| \leq 1$ or $V_{j} \subseteq S_{i}$

Proof. Let us consider the smallest $i$ for which the property is not true. Assume that only some vertices of the copy set $V_{j}$ are added to $S_{i}$. Since $V_{j}$ is not complete, we can postpone adding the vertices of $V_{j}$ to the solution set. By induction, copy sets are added only completely to the solution set.

Assume now that a removal removes vertices from $V_{j}$ such that more than one vertex of $V_{j}$ remains in $S_{i}$. Then this happened since we added a complete copy set $V_{p}$ into the solution set. Thus, there are other copy sets $V_{j_{1}}, \ldots, V_{j_{x}}$ from which parts were removed from $S_{i}$. If one of the copy sets $V_{j^{*}}$ is never re-added completely (or if this only happens after a complete removal of $V_{j^{*}}$ ), then we can also remove this copy set completely instead of removing several copy sets partly. Otherwise, let $V_{j^{*}}$ be the copy set that is completely re-added last in a layer $i^{\prime}>i$. If the number of vertices from $W \cup S_{1}$ that are in the solution does not decrease from layer $i-1$ to layer $i$, then we can completely remove $V_{j^{*}}$ in layer $i$ and re-add it completely in layer $i^{\prime}$ in such a way that the solution in layer $i^{\prime}$ is again $S_{i^{\prime}}$. As any copy set which is completely contained in the solution in one layer is again completely contained in the solution of this layer, the solution remains valid. Moreover, it is not hard to see that the number of relocations does not increase by this change. (In layer $i$, both solutions perform $4 \tilde{k}$ changes. The new solution performs $4 \tilde{k}$ changes in layer $i^{\prime}$, while the old solution performs $4 \tilde{k}$ changes that the new solution does not perform to completely contain $V_{j_{1}}, \ldots, V_{j_{x}}$ at some point inside the layers $i+1, \ldots, i^{\prime}$.)

If the number of vertices from $W \cup S_{1}$ being in the solution decreases from layer $i-1$ to layer $i$, then we remove all except one vertex of $V_{j^{*}}$ together with the vertex from $W \cup S_{1}$ and add $V_{p}$ in layer $i$. We add the $4 \tilde{k}-1$ vertices of $V_{j^{*}}$ again in layer $i^{\prime}$ in such a way that the solution in layer $i^{\prime}$ is again $S_{i^{\prime}}$. Again, the number of relocations does not increase. (In layer $i$, both solutions perform $4 \tilde{k}$ changes. The new solution performs $4 \tilde{k}-1$ changes in layer $i^{\prime}$ as the last vertex of $V_{j^{*}}$ is not relocated between layer $i$ and $i^{\prime}$, but does not perform the $4 \tilde{k}-1$ changes to completely contain $V_{j_{1}}, \ldots, V_{j_{x}}$ at some point inside the layers $i+1, \ldots, i^{\prime}$.)

By applying this change exhaustively, this results in a solution fulfilling the desired property.

5. $S_{i} \backslash S_{i-1} \subseteq W$ for any $i \geq 3$.

Proof. Assume that the property is not true, i.e., some vertices of a complete copy set become part of $S_{i} \backslash S_{i-1}$ for some layer $i(i>2)$. Choose $i$ as large as possible such that a first vertex of a copy set $V_{j}$ becomes part of the solution, i.e., $S_{i-1} \cap V_{j}=\emptyset$ and $S_{i} \cap V_{j} \neq \emptyset$.

We can undo the whole relocation of the copy set, which saves $4 \tilde{k}$ relocations, but causes some additional relocations: In the worse case, we have to pay (1) for one more relocation in the current edge gadget (if the newly added copy set has a larger index than the removed one) and (2) one extra relocation in the last layer of $\tilde{k}-1$ edge gadgets. To see (2) observe that the new copy set can be combined with at most $\tilde{k}-1$ complete other copy sets in the current solution (Property 4 ) and so help us to save the extra relocation for $\tilde{k}-1$ edge gadgets.

6. Every solution for (all layers of) the edge gadget $\mathcal{G}_{\left\{v_{i}, v_{j}\right\}}$ needs at least $8 \tilde{k}^{2}(n-\tilde{k})$ relocations. Unless the copy sets $V_{i}$ and $V_{j}$ are in the solution strictly before the first layer of that gadget begins (good case), at least one additional extra relocation is necessary.

Proof. By Property 5 , we may assume that only center vertices are relocated. By choice 
of $k$, there are at most $\tilde{k}$ copy sets in the solution. Ignoring the last layer of the edge gadget, we thus must relocate the $8 \tilde{k}^{2}$ copies of the $n-\tilde{k}$ vertices whose copy sets are not in the solution. Furthermore the last layer of the edge gadget needs an extra relocation unless $V_{i}$ and $V_{j}$ are part of the solution.

It is easy to see that the reduction is computable in polynomial time. We now show the correctness of the two directions of the reduction.

- Lemma 14. If $H$ contains a clique of size $\tilde{k}$, then the Global Multistage Vertex COVER instance $(\mathcal{G}, k, \ell)$ admits a solution.

Proof. Let $C=\left\{v_{i_{1}}, v_{i_{2}}, \ldots, v_{i_{\tilde{k}}}\right\} \subseteq V(H)$ be a clique in $H$. We construct a solution $\mathcal{S}=\left(S_{1}, \ldots, S_{\tau}\right)$ for $(\mathcal{G}, k, \ell)$ as follows. In all layers except the first, the vertex cover contains the copy sets $V_{i_{j}}$ for all $j \in[\tilde{k}]$. We denote the set of these vertices by $S:=\bigcup_{j \in[\tilde{k}]} V_{i_{j}}$. Thus, we have used $k-1$ vertices in our solution. The remaining vertex in the solution, called jumping vertex, is the vertex $c_{i}$ in all layers $G_{i}$ in which $S$ is not a vertex cover (i.e., for every edge gadget, $j \in\left[8 \tilde{k}^{2}\right]$ and $i \in[n] \backslash\left\{i_{1}, \ldots, i_{\tilde{k}}\right\}$, the $(i+j n)$-th layer of the edge gadget, and the last layer of any edge gadget corresponding to an edge $\{v, w\}$ with $v \notin C)$. Otherwise, we do not change the solution, i.e., $S_{i}:=S_{i-1}$.

The temporal vertex set $\mathcal{S}$ is indeed a vertex cover of size $k$ in each layer. There are $4 \tilde{k}^{2}$ elocations from the first layer to the second layer. By Property 6 , the jumping vertex relocates in each $\mathcal{G}_{e}$ with $e$ belonging to the clique $8 \tilde{k}^{2}(n-\tilde{k})$ times, while it relocates $8 \tilde{k}^{2}(n-\tilde{k})+1$ times in all other edge gadgets. Thus, the total number of relocations is $4 \tilde{k}^{2}+8 m \tilde{k}^{2}(n-\tilde{k})+m-\left(\begin{array}{c}\tilde{k} \\ 2\end{array}\right)=\ell$.

It remains to show the reverse direction.

- Lemma 15. If the Global Multistage Vertex Cover instance $(\mathcal{G}, k, \ell)$ admits a solution, then $H$ contains a clique of size $\tilde{k}$.

Proof. Let us consider a solution that adds $\tilde{k}$ copy sets in $S_{2} \backslash S_{1}$ and adds no further copy sets in later layers (Properties 3 and 5 ). Furthermore, the edge gadget causes in total $8 m \tilde{k}^{2}(n-\tilde{k})$ relocations by Property 6 . Thus, $m-\left(\begin{array}{c}\tilde{k} \\ 2\end{array}\right)$ relocations remain from the $\ell$ allowed relocations. Thus there are $\left(\begin{array}{l}\tilde{k} \\ 2\end{array}\right)$ edge gadgets for which we do not pay an extra relocation due to the last layer. To save the extra relocation for that many edge gadgets, we must have an edge between all pairs of vertices whose copy sets are in the solution. To sum up, there is a clique of size $\tilde{k}$.

\subsubsection{Path Contraction}

Vertex gadget. For each $v_{i} \in V(H)$, we add a path of length $4 \tilde{k}$, and call the endpoints of the path $s_{i}$ and $t_{i}$. The edges of this path are the copy set $V_{i}$ of $v_{i}$.

Edge gadget. An edge gadget for an edge $e=\left\{v_{p}, v_{q}\right\}$ adds $8 \tilde{k}^{2} n+1$ layers. It adds $16 \tilde{k}^{2} n+4$ vertices $w_{e}^{1}, w_{e}^{2}, \ldots, w_{e}^{8 \tilde{k}^{2} n}, x_{e}^{1}, \ldots, x_{e}^{8 \tilde{k}^{2} n}, y_{e}^{1}, y_{e}^{2}$, and $z_{e}^{1}$ and $z_{e}^{2}$, where $w_{e}^{r}$ and $x_{e}^{r}$ $\left(r \in\left[8 \tilde{k}^{2} n\right]\right)$ are isolated in all but one layer of the gadget. For each $i \in[n]$ and $j \in\left[8 \tilde{k}^{2}\right]$, the edges $\left\{w_{e}^{i+j n}, s_{i}\right\}$ and $\left\{s_{i}, x_{e}^{i+j n}\right\}$ are present in the $(i+j n)$-th layer of the gadget. In the $\left(8 \tilde{k}^{2} n+1\right)$-th layer, the edges $\left\{t_{p}, t_{q}\right\},\left\{s_{p}, y_{e}^{1}\right\},\left\{y_{e}^{1}, y_{e}^{2}\right\},\left\{s_{p}, z_{e}^{1}\right\}$, and $\left\{z_{e}^{1}, z_{e}^{2}\right\}$ are present. Let $W$ be the set of edges incident to $w_{e}^{i}, x_{e}^{i}, y_{e}^{1}$, or $z_{e}^{1}$ for some edge $e$ and $i \in\left[8 \tilde{k}^{2} n\right]$. See Figure 2 for an example. 


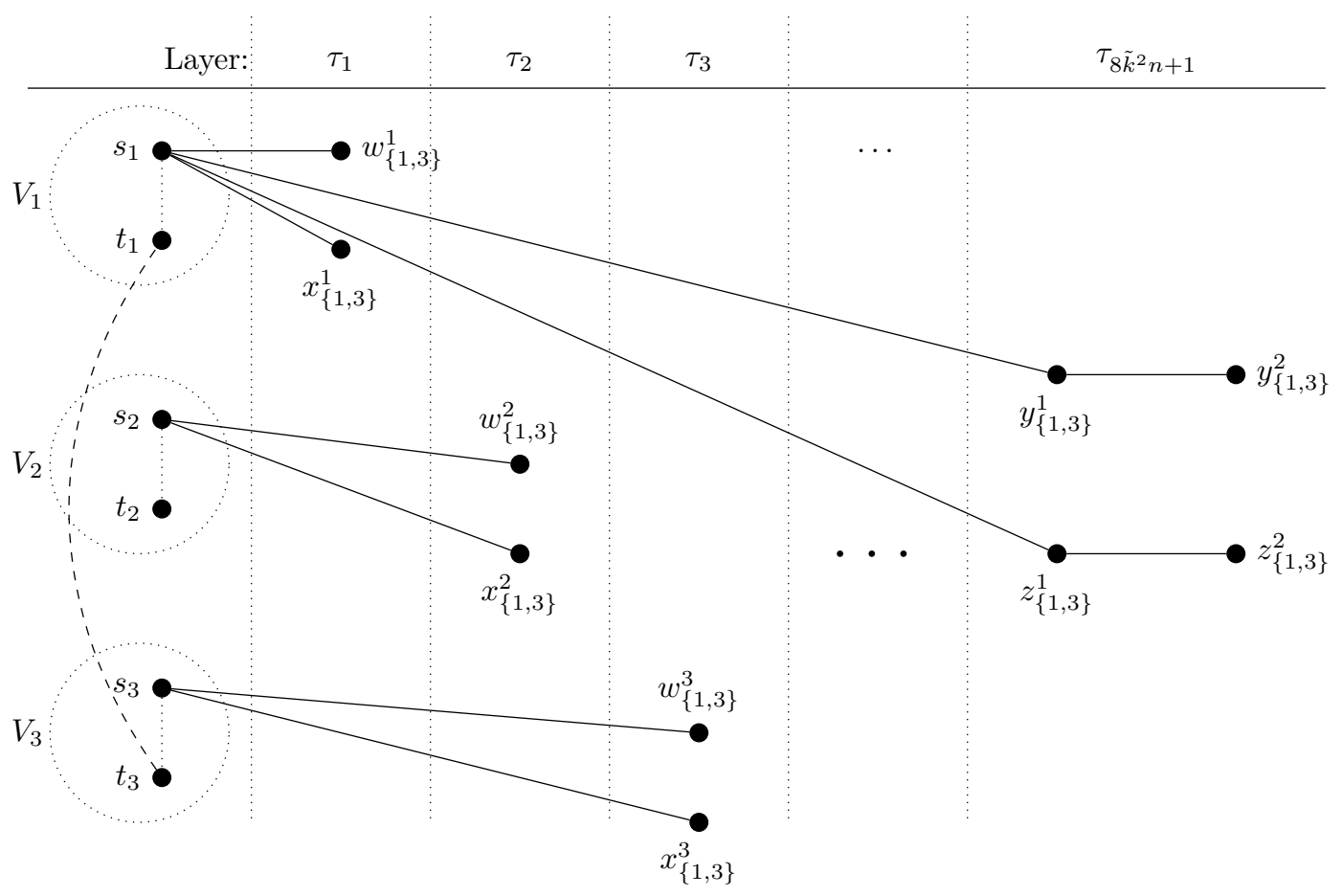

Figure 2 An example of the edge gadget for the edge $e=\left\{v_{1}, v_{3}\right\}$ in a graph with three vertices $v_{1}, v_{2}$, and $v_{3}$ for $\tilde{k}=3$. The $8 \tilde{k}^{2}$ repetitions of layers 1 to 3 (with different $x_{e}^{i}$ and $w_{e}^{i}$ ) are not drawn to keep the figure readable. The dotted edges represent a path of length $4 \tilde{k}^{2}$ (the copy set of the corresponding vertex). The edges are only contained in the layers in which the right end vertex $\left(w_{e}^{i}\right.$ or $\left.v_{e}\right)$ lies, with the exception of the edges inside a copy set, which are contained in all layers. The dashed edge is contained only in the last layer $\left(\tau_{8 \tilde{k}^{2} n+1}\right)$. The copy sets are encircled by dotted lines.

The edges $\left\{w^{i+j n}, s_{i}\right\}$ and $\left\{\left\{s_{p}, y_{e}^{1}\right\},\left\{y_{e}^{1}, y_{e}^{2}\right\}\right\}$ now correspond to the center vertices from the reduction for Global Multistage Vertex Cover (meaning that these edges will be added to the solution using relocations for layers not corresponding to edges or vertices of the clique).

Instance. Before all edge gadgets, in a first layer we have a clique of size $4 \tilde{k}^{2}+4$, ensuring that the solution in the first layer consists of $4 \tilde{k}^{2}+2$ edges of these paths. The vertices of this clique are isolated in all other layers. Afterwards, there are $m$ edge gadgets, one for each edge of the graph $H$. We set $k=4 \tilde{k}^{2}+2$ and $\ell=4 \tilde{k}^{2}+8 m \tilde{k}^{2}(n-\tilde{k})+\left(2 m-\left(\begin{array}{c}\tilde{k} \\ 2\end{array}\right)\right)$.

Note that this reduction and the previous reduction are very similar. Property 1 Property 5 are valid also for the current reduction (with $\left|S_{i} \cap W\right| \leq 2$ in Property 2 and $\left|S_{1} \cap S_{i}\right| \leq 2$ in Property 4). Moreover, Property 6 for an edge gadget of edge $\{i, j\}$ is also valid if we increment the number of relocations for the good case by one from $8 \tilde{k}^{2}(n-\tilde{k})$ to $8 \tilde{k}^{2}(n-\tilde{k})+1$. As before, if we are not in the good case, then we have one extra relocation since the last layer cause an extra relocation exactly if one of the copy sets $V_{i}$ and $V_{j}$ is not in the solution set. To see this, note that the last layer of the gadget forms a star whose center is the endpoint of three paths. Two are of length two and one is either very long (if $V_{i}$ or $V_{j}$ is not in the solution set) or of length 1 . To find a solution, a shortest path has to be contracted. 
It is easy to see that the reduction is computable in polynomial time. By replacing "vertex cover" through "path contraction" and $8 \tilde{k}^{2}(n-\tilde{k})$ by $8 \tilde{k}^{2}(n-\tilde{k})+1$ in the proofs of Lemmas 14 and 15 , we obtain a proof that shows the following: $H$ contains a clique of size $\tilde{k}$ exactly if the Global Multistage Path Contraction instance $(\mathcal{G}, k, \ell)$ admits a solution.

\subsubsection{Cluster Edge Deletion}

We now show W[1]-hardness with respect to solution size $k$ for Global Multistage Cluster Edge Deletion. Recall that, for a graph $H$, a set $D \subseteq E(H)$ is called a cluster edge deletion set if $H-D$ is a cluster graph (i.e., all connected components are cliques). We again reduce from Clique. Let $(H, \tilde{k})$ be an instance of CliquE. We construct a temporal graph $\mathcal{G}$ as follows.

Vertex gadget. For each vertex $v_{i} \in V(H)$, we add a clique of size $4 \tilde{k}+1$ containing a special vertex $u_{i}$. The edges adjacent to $u_{i}$ in this clique are the copy set of $v_{i}$.

Edge gadget. An edge gadget for an edge $e=\left\{v_{p}, v_{q}\right\}$ adds $8 \tilde{k}^{2} n+1$ layers. It adds $8 \tilde{k}^{2} n$ vertices $w_{e}^{1}, w_{e}^{2}, \ldots, w_{e}^{8 \tilde{k}^{2} n}$, which are all isolated in all but one layer. In the $(i+j n)$-th $\left(j \in\left[8 \tilde{k}^{2}\right]\right)$ layer of the gadget, vertex $w_{e}^{i+j n}$ is connected to $u_{i}$. In the $\left(8 \tilde{k}^{2} n+1\right)$-th layer, the edge $\left\{u_{p}, u_{q}\right\}$ is present. See Figure 3 for an example.

The edges $\left\{w_{e}^{i+j n}, u_{i}\right\}$ and $\left\{u_{p}, u_{q}\right\}$ correspond to the center vertices from the reduction for Global Multistage Vertex Cover.

Instance. In the first layer of the temporal graph, we have $4 \tilde{k}^{2}+1$ vertex-disjoint paths of length two, ensuring that the solution in the first layer consists of $4 \tilde{k}^{2}+1$ edges of these paths. The vertices of the paths are isolated in all other layers. Afterwards, there are $m$ edge gadgets, one for each edge of graph $H$. We set $k=4 \tilde{k}^{2}+1$ and $\ell=8 m \tilde{k}^{2}(n-\tilde{k})+4 \tilde{k}^{2}+\left(m-\left(\begin{array}{c}\tilde{k} \\ 2\end{array}\right)\right)$.

Note that this and the reduction for Global Multistage Vertex Cover are very similar. Property 1 -Property 5 are valid also for the current reduction. The proof of Property 4 is even simpler, as any solution either contains no edge of a copy set for a vertex $v_{i}$, or it separates $u_{i}$ from the rest of the clique. This separation can be done without loss of generality by deleting the edges from the copy set. Moreover, Property 6 is also valid for all edge gadgets. As before, if we are not in the good case, we have one extra relocation since the last layer causes an extra relocation exactly if one of the copy sets $V_{i}$ and $V_{j}$ is not in the solution set. To see this, note that the last layer of the gadget forms two cliques connected by the edge $\left\{u_{p}, u_{q}\right\}$. To find a solution, the edge $\left\{u_{p}, u_{q}\right\}$ has to be deleted, or the vertices $u_{p}$ and $u_{q}$ have to be separated from the clique they are contained in, which needs at least $4 \tilde{k}$ edges and can be done by deleting the copy set of these vertices.

It is easy to see that the reduction is computable in polynomial time. Replacing "vertex cover" by "cluster edge deletion set" in the proofs of Lemmas 14 and 15 we obtain a proof showing that $H$ contains a clique of size $\tilde{k}$ exactly if the Global Multistage Cluster EDGE DeLETION instance $(\mathcal{G}, k, \ell)$ admits a solution.

We remark that the reduction for Global Multistage Cluster Edge Deletion also yields hardness for Global Multistage Cluster Editing. 


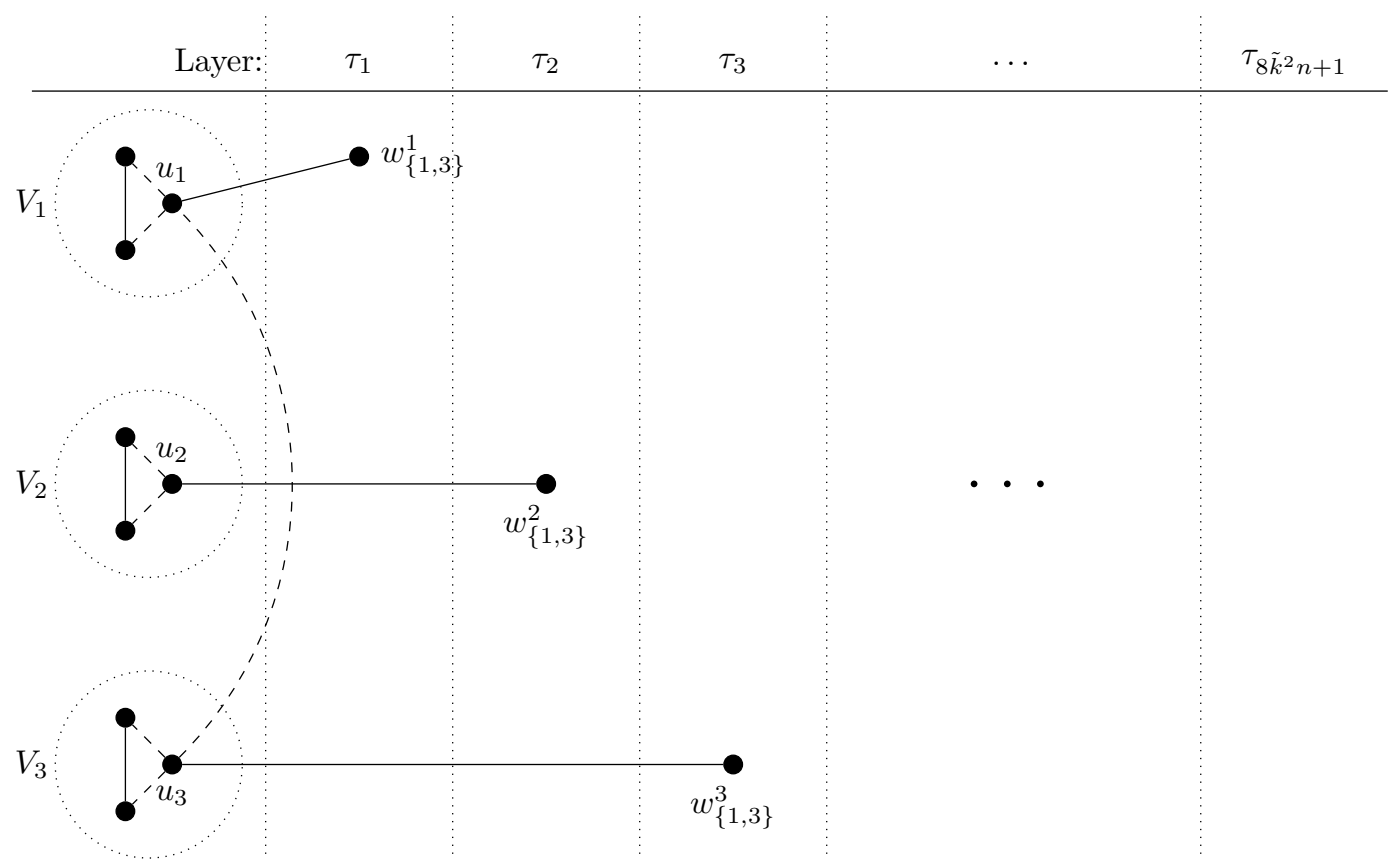

Figure 3 An example of the edge gadget for the edge $e=\left\{v_{1}, v_{3}\right\}$ in a graph with three vertices $v_{1}, v_{2}$, and $v_{3}$ for $\tilde{k}=3$. The $8 \tilde{k}^{2}$ repetitions of layers 1 to 3 (with different center vertices!) are not drawn to keep the figure readable. Furthermore, each copy set contains only two instead of twelve edges. The edges are only contained in the layer in which the right end vertex $\left(w_{e}^{i}\right.$ or $\left.w_{e}^{*}\right)$ lies, except for the long dashed edge, which is only present in layer $8 \tilde{k}^{2} n+1$. The copy set $V_{i}$ consists of the short dashed edges contained in the corresponding dotted circle.

\subsection{Hardness for the Combined Parameter $k+\ell$}

The problems investigated in the previous section are all superset-enumerable and, therefore, their global multistage variants admit FPT-algorithms parameterized by $k+\ell$. However, for some classical, monotone graph problems no superset-enumeration by solution size is known. Two notable examples are Planar Dominating Set and Planar Edge Dominating SET, which in their classic (static) variants are known to be fixed-parameter tractable for the parameter solution size. Assuming FPT $\neq \mathrm{W}[2]$, we will that these problems are not superset-enumerable and do not admit FPT algorithms for the their global multistage versions by proving that these are both W[2]-hard for parameter $k$ even if $\ell=0$. Note that this also implies $\mathrm{W}[1]-/ \mathrm{W}[2]$-hardness for the classical multistage version of these problems.

\subsubsection{Planar Dominating Set}

Recall that, for a graph $H$, a set $D \subseteq V(H)$ is called a dominating set if $D \cup \bigcup_{d \in D} N(d)=$ $V(H)$.

$\triangleright$ Problem (Global Multistage Planar Dominating Set). Given a temporal graph $\mathcal{G}=\left\langle G_{1}, \ldots, G_{\tau}\right\rangle$ that is planar in every time step and parameters $k, \ell$, find sets $S_{1}, \ldots, S_{\tau}$, each of size at most $k$, such that $S_{i} \subseteq V\left(G_{i}\right)$ is a dominating set for $G_{i}(i \in\{1, \ldots, \tau\})$ and the total number of insertions $\sum_{i}\left|S_{i+1} \backslash S_{i}\right|$ is at most $\ell$.

- Proposition 16. Global Multistage Planar Dominating Set is W[2]-hard when parameterized by the solution size $k$ even when no relocations are allowed $(\ell=0)$. 

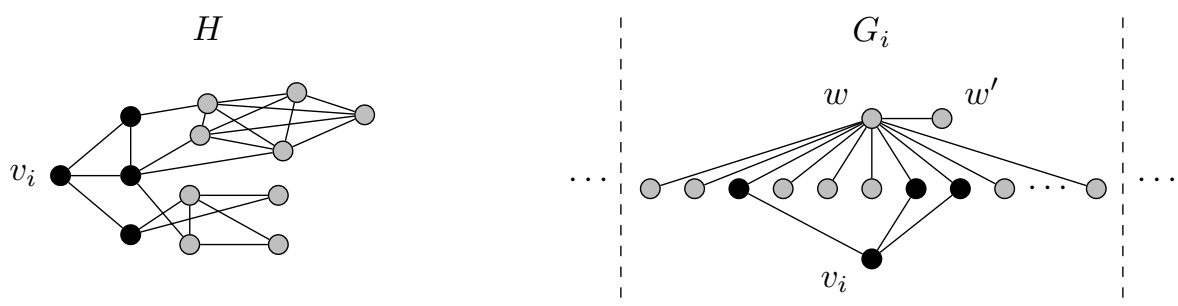

Figure 4 For each vertex $v_{i}$ in the graph $H$, a graph $G_{i}$ is constructed where vertex $w$ is connected to all vertices except for $v_{i}$, and $v_{i}$ is connected only to its neighbors in $H$.

Proof. We provide a parameterized reduction from the W[2]-hard Dominating SET problem [13]. Given a Dominating Set instance $\mathcal{I}=\left(H, k^{\prime}\right)$, we construct an instance $\mathcal{I}^{\prime}=(\mathcal{G}, k, \ell=0)$ of Global Multistage Planar Dominating set as follows.

Let $w$ and $w^{\prime}$ be two new vertices. For each vertex $v_{i} \in V(H)(i=1, \ldots, n)$ create a time layer $G_{i}$ with $V\left(G_{i}\right)=V(H) \cup\left\{w, w^{\prime}\right\}$ and $E\left(G_{i}\right)=\left\{\left\{v_{i}, u\right\} \mid \forall u \in N\left(v_{i}\right)\right\} \cup\{\{w, u\} \mid \forall u \in$ $\left.V(H) \backslash\left\{v_{i}\right\}\right\} \cup\left\{\left\{w, w^{\prime}\right\}\right\}$. For an example see Figure 4 Observe that in every time step $i \in\{1, \ldots, n\}, G_{i}$ without $v_{i}$ forms a star and connecting $v_{i}$ with some vertices of the star preserves planarity.

We claim that $H$ contains a dominating set $D$ of size $k$ if and only if $\mathcal{G}$ contains a multistage dominating set $D^{\prime}$ of size $k+1$. For the forward direction, choose $D^{\prime}=D \cup\{w\}$. For every time step $i$, vertex $v_{i}$ is dominated in $G_{i}$ by a vertex in $D$. All other vertices are dominated in $G_{i}$ by vertex $w$. For the reverse direction, we have to dominate $w^{\prime}$ in each time step. Without loss of generality, we can assume that $w \in D^{\prime}$. Then $D \backslash\left\{w^{\prime}\right\}$ must be a dominating set in $H$ since every vertex $v_{i}$ is, at time step $i$, only adjacent to its neighbors from $H$.

\subsubsection{Planar Edge Dominating Set}

For a graph $H, D \subseteq E(H)$ is called an edge dominating set if each edge of $H$ is incident to an edge in $D$. Edge Dominating Set, the problem of finding a minimum-size edge dominating set, is closely related to VerTex Cover and Matching. However, even when restricting Edge Dominating Set to planar graphs, within the global multistage setting it appears to be computationally harder than VERTEX COVER on general graphs, as we will show now. The restriction of Global Multistage Edge Dominating Set, which we call Global Multistage Fully Planar Edge Dominating Set, is stronger than the one of Global Multistage Planar Dominating Set, as we now require the underlying graph to be planar, while we only required every layer to be planar for Global Multistage Planar Dominating Set.

$\triangleright$ Problem (Global Multistage Fully Planar Edge Dominating Set). Given a triple $(\mathcal{G}, k, \ell)$ where $\mathcal{G}=\left\langle G_{1}, \ldots, G_{\tau}\right\rangle$ is a temporal graph with planar underlying graph $G$, the goal is to find sets $S_{1}, \ldots, S_{\tau}$, each of size at most $k$, such that $S_{i} \subseteq E(G)$ is an edge dominating set for $G_{i}(i \in[\tau])$ and the number of insertions $\sum_{i}\left|S_{i+1} \backslash S_{i}\right|$ is at most $\ell$.

- Proposition 17. Global Multistage Fully Planar Edge Dominating Set is W[2]hard when parameterized by the solution size $k$ and no relocations are allowed $(\ell=0)$.

Proof. We will devise a parameterized reduction from SET Cover here, which is well-known to be W[2]-hard [13]. A SET Cover instance consists of an integer $n$ and a family $\mathcal{F} \subseteq 2^{[n]}$ 


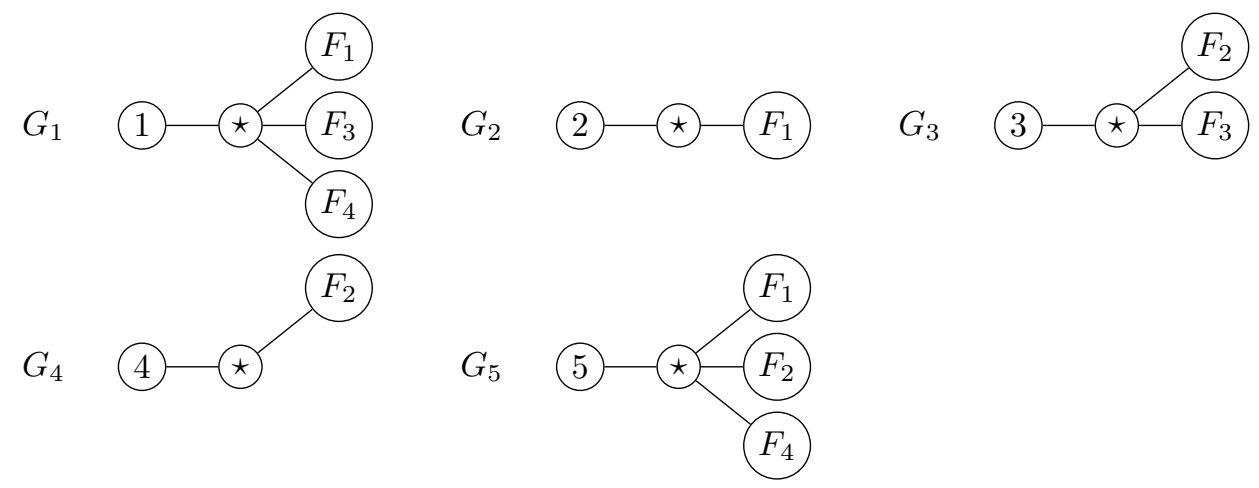

Figure 5 An example of a temporal graph $\mathcal{G}=\left\langle G_{1}, \ldots, G_{5}\right\rangle$ for the parameterized reduction from Set Cover to Global Multistage Fully Planar Edge Dominating Set using the family $\mathcal{F}=\left\{F_{1}=\{1,2,5\}, F_{2}=\{3,4,5\}, F_{3}=\{1,3\}, F_{4}=\{1,5\}\right\}$. Isolated vertices are not shown.

of subsets of $[n]$. The task is to find a subset $\mathcal{S} \subseteq \mathcal{F}$ of size at most $\tilde{k}$ such that $\bigcup \mathcal{S}=[n]$. We assume without loss of generality that $\bigcup \mathcal{F}=[n]$ and $\emptyset \notin \mathcal{F}$.

From this, we construct a temporal graph as follows. Let $V:=\{\star\} \cup[n] \cup \mathcal{F}$ be the set of vertices. At time step $i \in[n]$, we define the edge set as $E\left(G_{i}\right):=\{\{\star, i\},\{\star, F\}: F \in \mathcal{F} \wedge i \in$ $F\}$. For an example see Figure 5 Note that the underlying graph $G$ is then a star with center vertex $\star$. We claim that $\mathcal{G}$ contains an edge dominating set of size $k$ if and only if the SET Cover instance has a solution of size $\tilde{k}$.

We may assume that any solution to Global Multistage Fully Planar Edge Dominating SET only contains edges of the form $\{\star, F\}$ with $F \in \mathcal{F}$ since any other edge may be exchanged for one of these.

Now the claimed equivalence follows from the fact that $\mathcal{F}^{\prime} \subseteq \mathcal{F}$ is a set cover if and only if $\left\{\{\star, F\} \mid F \in \mathcal{F}^{\prime}\right\}$ is an edge dominating set for each layer of $\mathcal{G}$.

In summary, we have shown that the temporal multistage version of Dominating SET is $\mathrm{W}[2]$-hard for the parameter $k+\ell$ even when each layer is a planar graph for Dominating SET or when the underlying graph is a star for Edge Dominating SET.

\section{Parameterized Hardness of Polynomial-Time Solvable Problems Gone Globally Multistage}

We now present parameterized hardness results for the global multistage versions of several polynomial-time solvable problems. These hold even if no relocations are allowed $(\ell=0)$ and thus also imply parameterized hardness for the corresponding classical multistage versions of these problems.

\section{$5.1 s-t$-Path}

Contrasting very recent work on $s$-t-PATH in the standard multistage setting [17, we show that the global multistage version of $s$ - $t$-PATH with no relocations is W[1]-hard parameterized by the combined parameter solution size $k$ plus lifetime $\tau$. The problem is formally defined as follows.

$\triangleright$ Problem (Global Multistage $s$ - $t$-Path). Given a temporal graph $\mathcal{G}=\left\langle G_{1}, \ldots, G_{\tau}\right\rangle$, two vertices $s, t \in V(G)$, and an integer $k$, find a set $F \subseteq E(G)$ of at most $k$ edges such that, 


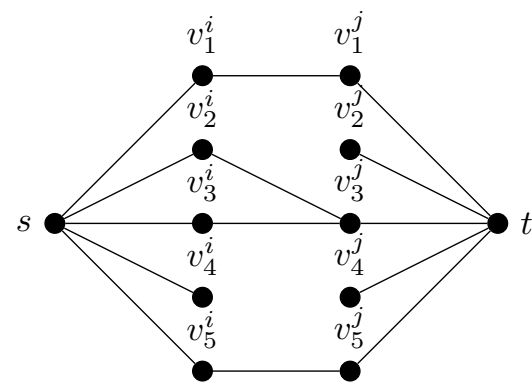

a) Global Multistage $s$ - $t$-Path.

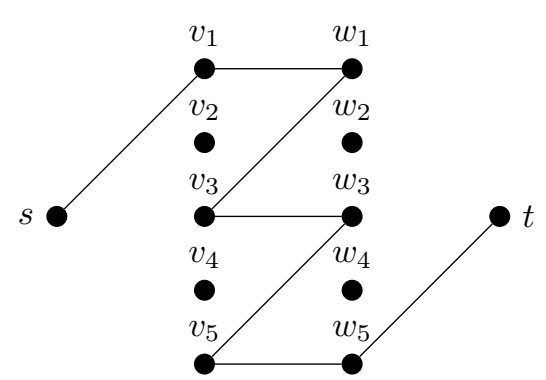

b) Global Multistage $s$ - $t$-Cut.

Figure 6 a) An example for the layer $L_{i j}$ for the reduction to Global Multistage $s$ - $t$-Path, where $V_{i}=\left\{v_{1}^{i}, \ldots, v_{5}^{i}\right\}, V_{j}=\left\{v_{1}^{j}, \ldots, v_{5}^{j}\right\}$, and $E\left(H\left[V_{i} \cup V_{j}\right]\right)=\left\{\left\{v_{1}^{i}, v_{1}^{j}\right\},\left\{v_{2}^{i}, v_{3}^{j}\right\},\left\{v_{3}^{i}, v_{3}^{j}\right\},\left\{v_{5}^{i}, v_{5}^{j}\right\}\right\}$. b) An example for the $j$-th layer of $\mathcal{G}$ for the reduction to Global Multistage $s$ - $t$-Cut for a subset $S_{j}=\left\{u_{1}, u_{3}, u_{5}\right\} \subseteq U$ with $U=\left\{u_{1}, u_{2}, u_{3}, u_{4}, u_{5}\right\}$.

for every $i \in[\tau]$, there exists an $s$-t-path in $G_{i}^{\prime}:=\left(V\left(G_{i}\right), E\left(G_{i}\right) \cap F\right)$.

- Theorem 18. Global Multistage $s$ - $t$-Path parameterized by $k+\tau$ is W[1]-hard, even if $\ell=0$.

Proof. We give a parameterized reduction from the W[1]-hard Multicolored Clique problem [13] In this problem, a graph $H$ with a partition $\left(V_{1}, \ldots, V_{k^{\prime}}\right)$ of $V(H)$ into $k^{\prime}$ sets is given, and the question is whether $H$ contains a clique containing exactly one vertex from $H_{i}$ for all $i \in\left[k^{\prime}\right]$.

Let $\left(H, k^{\prime}, c\right)$ be an instance of Multicolored Clique where $c: V(H) \rightarrow\left[k^{\prime}\right]$ is the coloring determining a $k^{\prime}$-partition $V_{1}, \ldots, V_{k^{\prime}}$ of $V(H)$. We define a temporal graph $\mathcal{G}$ with vertex set $V(\mathcal{G}):=V(H) \cup\{s, t\}$ as follows (see also Figure 6a). For each $i \in\left[k^{\prime}\right]$, the $i$ th layer of $\mathcal{G}$ contains the edges $\{s, v\}$ and $\{v, t\}$ for each $v \in V_{i}$. For $i, j \in\left[k^{\prime}\right]$ with $i<j$, we add a layer $L_{i, j}$, which contains the edges $\{s, v\}$ for all $v \in V_{i}$, the edges from $E\left(H\left[V_{i} \cup V_{j}\right]\right)$, and the edges $\{v, t\}$ for all $v \in V_{j}$. We set $k:=2 k^{\prime}+\left(\begin{array}{c}k^{\prime} \\ 2\end{array}\right)$ and claim that the resulting Global Multistage $s$ - $t$-Path instance $(\mathcal{G}, k, 0)$ is solvable if and only if $H$ contains a multicolored $k^{\prime}$-clique.

$(\Leftarrow)$ Given a clique $\left\{v_{1}, \ldots, v_{k^{\prime}}\right\}$ with $v_{i} \in V_{i}$, we construct a solution for $(\mathcal{G}, k, 0)$ by taking the edges $\left\{s, v_{i}\right\},\left\{v_{i}, t\right\}$, and $\left\{v_{i}, v_{j}\right\}$ for all $i, j \in\left[k^{\prime}\right]$. For $i \in\left[k^{\prime}\right]$, the $i$ th layer contains the $s$-t-path $s$ - $v_{i}-t$. The layer $L_{i, j}$ contains the $s$-t-path $s-v_{i}-v_{j}-t$.

$(\Rightarrow)$ Given a solution to $(\mathcal{G}, k, 0)$, note that the first $k^{\prime}$ layers require that for each $i \in\left[k^{\prime}\right]$, there is some $v_{i} \in V_{i}$ such that $\left\{s, v_{i}\right\}$ and $\left\{v_{i}, t\right\}$ are contained in the solution. The layer $L_{i, j}$ for $i<j$ requires that an edge $e=\left\{v_{i j}, w_{i j}\right\}$ with $v_{i j} \in V_{i}$ and $w_{i j} \in V_{j}$ from $E\left(H\left[V_{i} \cup V_{j}\right]\right)$ is contained in the solution, and that the edges $\left\{s, v_{i j}\right\}$ and $\left\{w_{i j}, t\right\}$ are also contained in the solution. Since $k=2 k^{\prime}+\left(\begin{array}{c}k^{\prime} \\ 2\end{array}\right)$, no edge but $\left\{s, v_{i}\right\},\left\{v_{i}, t\right\}$, and $\left\{v_{i j}, w_{i j}\right\}$ for $i, j \in\left[k^{\prime}\right]$ with $i<j$ is contained in the solution. We can conclude that $v_{i j}=v_{i}$ and $w_{i j}=v_{j}$ for all $i, j \in\left[k^{\prime}\right]$ with $i<j$, implying that $\left\{v_{1}, \ldots, v_{k^{\prime}}\right\}$ is a clique.

The W[1]-hardness for GLOBAL Multistage $s$ - $t$-PATH follows directly since $k=2 k^{\prime}+\left(\begin{array}{c}k^{\prime} \\ 2\end{array}\right)$ and $\tau=k^{\prime}+\left(\begin{array}{c}k^{\prime} \\ 2\end{array}\right)$. 


\section{$5.2 s-t-$ Cut}

We now consider the problem of separating the vertices $s$ and $t$ in each layer. We show that the global multistage version of $s$-t-CuT with no relocations problem is W[2]-hard parameterized by solution size $k$.

$\triangleright$ Problem (Global Multistage $s$ - $t$-Cut). Given a temporal graph $\mathcal{G}=\left\langle G_{1}, \ldots, G_{\tau}\right\rangle$, two vertices $s$ and $t$, and an integer $k$, decide whether there exists a set $F \subseteq E(\mathcal{G})$ of at most $k$ edges such that, for every $i \in[\tau]$, there exists no $s$ - $t$-path in $G_{i}^{\prime}:=\left(V\left(G_{i}\right), E\left(G_{i}\right) \backslash F\right)$. The set $F$ is called a temporal s-t-cut.

We show that this problem is W[2]-hard by a parameterized reduction similar to the one for Global Multistage $s$ - $t$-Path.

- Theorem 19. Global Multistage $s$-t-Cut parameterized by $k$ is W[2]-hard, even if $\ell=0$.

Proof. We give a parameterized reduction from the W[2]-hard HitTing SET problem [13]. Here we are given a set $U$, a set familiy $\mathcal{S} \subseteq 2^{U}$, and an integer $k^{\prime}$ and need to find a subset of at most $k^{\prime}$ elements of $U$ which intersects every member of $\mathcal{S}$.

Given an instance $\mathcal{I}=\left(U, \mathcal{S}, k^{\prime}\right)$ of Hitting SET, we design an instance of GlobAL Multistage $s$ - $t$-Cut as follows (see also Figure 6b). The graph $\mathcal{G}$ contains two vertices $s$ and $t$. Furthermore, for each $u_{i} \in U$, there are two vertices $v_{i}$ and $w_{i}$. For each set $S_{j}=$ $\left\{u_{i_{1}}, u_{i_{2}}, \ldots, u_{i_{r}}\right\} \in \mathcal{S}$, the $j$-th layer contains the $s$ - $t$-path $s-v_{i_{1}}-w_{i_{1}}-v_{i_{2}}-w_{i_{2}}-v_{i_{3}}-\ldots-v_{i_{r}}-w_{i_{r}}-t$. We set $k:=k^{\prime}$. We claim that the resulting Global Multistage $s$ - $t$-Cut instance $(\mathcal{G}, k, 0)$ is equivalent to the given Hitting $\operatorname{Set}$ instance $\left(U, \mathcal{S}, k^{\prime}\right)$.

$(\Leftarrow)$ Let $X=\left\{u_{i_{1}}, u_{i_{2}}, \ldots, u_{i_{k}}\right\}$ be a hitting set. We define $F:=\left\{\left\{v_{i_{j}}, w_{i_{j}}\right\}: j \in[k]\right\}$, and claim that $F$ separates $s$ and $t$ in each layer.

Consider the $j$-th layer of $\mathcal{G}$. This layer contains a unique $s$ - $t$-path, which contains the edge $\left\{v_{i}, w_{i}\right\}$ for each $u_{i} \in S_{j}$. As $X$ is a hitting set, there is an edge of this path contained in $F$ and, thus, $F$ is a temporal $s$-t-cut.

$(\Rightarrow)$ Let $F$ separate $s$ and $t$ in each layer and let $|F| \leq k$. If $F$ contains an edge of the form $\left\{s, v_{i}\right\},\left\{v_{i}, w_{j}\right\}$ for $j \neq i$, or $\left\{w_{i}, t\right\}$, then we can replace this edge by $\left\{v_{i}, w_{i}\right\}$, as any $s$ - $t$-path containing such an edge contains also the edge $\left\{v_{i}, w_{i}\right\}$. Thus, we assume that $F=\left\{\left\{v_{i}, w_{i}\right\}: i \in I\right\}$ for some $I \subseteq[n]$ with $|I|=k$.

Let $X:=\left\{u_{i}: i \in I\right\}$. Clearly, $|X|=|I|=k$, so it remains to show that $X$ is a hitting set. Consider a set $S_{j}$. Since $F$ is an $s$-t-cut in $G_{j}$, it contains an edge $e=\left\{v_{i}, w_{i}\right\}$ of the unique $s$ - $t$-path in this layer. Thus, we have $u_{i} \in X \cap S_{j}$ and, therefore, $X$ hits $S_{j}$.

The W[2]-hardness for Global Multistage $s$ - $t$-Cut follows directly since $k=k^{\prime}$.

\subsection{Matching}

Finally, we show that the global multistage version of MATCHING with no relocations is W[1]-hard parameterized by solution size $k$. This stands in contrast to recent fixed-parameter tractability results for a (non-multistage) temporal version of MATCHING based on time windows [6, 26]. The problem is formally defined as follows.

$\triangleright$ Problem (Global Multistage Matching). Given a temporal graph $\mathcal{G}=\left\langle G_{1}, \ldots, G_{\tau}\right\rangle$, and an integer $k$, decide whether there exists a set $F \subseteq E(\mathcal{G})$ of at least $k$ edges such that, for every $i \in[\tau]$, the elements of $E\left(G_{i}\right) \cap F$ are pairwise disjoint. The set $F$ is called a temporal matching. 


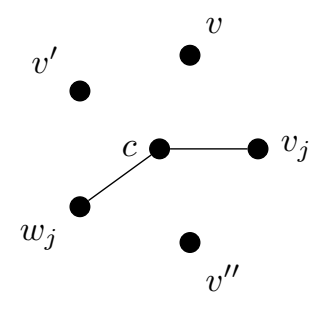

Figure 7 An example for the $i$-th layer of $\mathcal{G}$ for the Global Multistage Matching reduction from IndEPENDEnt Set for an edge $e_{i}=\left\{v_{i}, w_{i}\right\}$ and $V(H)=\left\{v_{i}, w_{i}, v, v^{\prime}, v^{\prime \prime}\right\}$.

Theorem 20. Global Multistage Matching parameterized by $k$ is W[1]-hard, even if $\ell=0$.

Proof. We give a parameterized reduction from the INDEPENDENT SET problem. Given a graph $H$ and an integer $k^{\prime}$, the problem INDEPENDENT SET asks whether $H$ contains a set of pairwise non-adjacent vertices of size at least $k^{\prime}$. It is well-known to be W[1]-hard parameterized by solution size [13].

Given an instance $\left(H, k^{\prime}\right)$ of INDEPENDENT SET, we construct an instance of GLOBAL Multistage Matching as follows. Let $E(H)=:\left\{e_{1}, e_{2}, \ldots, e_{m}\right\}$ with $e_{i}=:\left\{v_{i}, w_{i}\right\}$. The vertices of $\mathcal{G}$ are $V(\mathcal{G}):=V(H) \cup\{c\}$, where $c$ is not contained in $V(H)$. The graph $\mathcal{G}$ has $m$ layers. The $i$-th layer of $\mathcal{G}$ contains the edges $\left\{v_{i}, c\right\}$ and $\left\{w_{i}, c\right\}$, see Figure 7 for an example. Finally, we set $k:=k^{\prime}$.

We claim that the resulting Global Multistage Matching instance $(\mathcal{G}, k, 0)$ is equivalent to the INDEPENDENT SET instance $\left(H, k^{\prime}\right)$.

$(\Leftarrow)$ Let $X$ be an independent set in $H$. Define $M:=\{\{v, c\}: v \in X\}$. Clearly, $|M|=k$, so it remains to show that $M \cap E\left(G_{i}\right)$ is a matching for all $i \in[\tau]$. Every layer containing an edge $\left\{v_{i}, c\right\}$ contains only one other edge, namely $\left\{w_{i}, c\right\}$. If $v_{i} \in X$ ( $w_{i} \in X$ is symmetric), then $w_{i} \notin X$, as $X$ is an independet set. Thus, $F \cap E\left(G_{i}\right)=\left\{\left\{v_{i}, c\right\}\right\}$ is a matching. If neither $v_{i} \in X$ nor $w_{i} \in X$, then $F \cap E\left(G_{i}\right)=\emptyset$ is a matching.

$(\Rightarrow)$ Let $F \subseteq E(\mathcal{G})$ be a temporal matching of size $k$. Define $X:=\{v \in V(H):\{v, c\} \in F\}$. Clearly, $|X|=k$. It remains to show that $X$ is an independent set. So assume that $e_{i} \in E(H[X])$. Then $\left\{v_{i}, c\right\} \in F \cap E\left(G_{i}\right)$ and $\left\{w_{i}, c\right\} \in F \cap E\left(G_{i}\right)$, contradicting the assumption that $F$ is a temporal matching.

Now the W[1]-hardness for Global Multistage Matching follows directly since $k^{\prime}=k$.

\section{Conclusion}

We described a general approach to derive fixed-parameter tractability results (including polynomial-size problem kernels) for global multistage versions of classical NP-hard problems. A particular technical feature herein is showing how to derive FPT-algorithms for global multistage problems from FPT-enumeration algorithms for their static counterpart and "temporal kernels" from known static ones (more specifically, known full kernels [12]—a method that to the best of our knowledge has not yet been used in the design of algorithms for temporal (graph) problems). Our results are complemented by several parameterized hardness results, indicating that the parameter solution size alone does not suffice to obtain fixed-parameter tractability results. Furthermore, we could show that for some problems which do not fit our framework, the global multistage versions are hard in a parameterized 
sense, even though they are polynomial-time solvable in the static case. We remark that all our hardness results hold for the variant of the global multistage scenario where the first solution $S_{1}$ is given as part of the input.

As to challenges for future work, we also refer to the open questions exhibited in Table 1. In particular, the parameterized complexity with respect to the combined parameter $\tau+k$ is still open for some of the problems studied. From a more general perspective, exploring connections to dynamic problems [1, 19, 24, 25] and reoptimization [7] may be fruitful as well. Finally, studying (further) temporal problems in the global multistage setting may be of general interest in the area of complex network analysis.

\section{References}

1 Faisal N. Abu-Khzam, Judith Egan, Michael R. Fellows, Frances A. Rosamond, and Peter Shaw. On the parameterized complexity of dynamic problems. Theor. Comput. Sci., 607:426-434, 2015. doi:10.1016/j.tcs.2015.06.053. 2, 24

2 Evripidis Bampis, Bruno Escoffier, and Alexander Kononov. LP-based algorithms for multistage minimization problems, 2019. arXiv:1909.10354, 3, 4

3 Evripidis Bampis, Bruno Escoffier, Michael Lampis, and Vangelis Th. Paschos. Multistage matchings. In Proceedings of 16th Scandinavian Symposium and Workshops on Algorithm Theory (SWAT 2018), pages 7:1-7:13. Schloss Dagstuhl - Leibniz-Zentrum für Informatik, 2018. doi:10.4230/LIPIcs.SWAT.2018.7. 2,3

4 Evripidis Bampis, Bruno Escoffier, Kevin Schewior, and Alexandre Teiller. Online multistage subset maximization problems. In Proceedings of the 27th Annual European Symposium on Algorithms (ESA 2019), pages 11:1-11:14, 2019. doi:10.4230/LIPIcs. ESA.2019.11, 2]

5 Evripidis Bampis, Bruno Escoffier, and Alexandre Teiller. Multistage knapsack. In Proceedings of the 44th International Symposium on Mathematical Foundations of Computer Science (MFCS 2019), pages 22:1-22:14, 2019. doi:10.4230/LIPIcs.MFCS.2019.22, 2 , 3

6 Julien Baste, Binh-Minh Bui-Xuan, and Antoine Roux. Temporal matching. Theor. Comput. Sci., 806:184-196, 2020. doi:10.1016/j.tcs.2019.03.026. 22

7 Hans-Joachim Böckenhauer, Elisabet Burjons, Martin Raszyk, and Peter Rossmanith. Reoptimization of parameterized problems, 2018. arXiv:1809.10578, 2, 24

8 Sebastian Böcker, Sebastian Briesemeister, Quang Bao Anh Bui, and Anke Truß. Going weighted: Parameterized algorithms for cluster editing. Theor. Comput. Sci., 410(52):54675480, 2009. doi:10.1016/j.tcs.2009.05.006. 9

9 Robert Bredereck, Till Fluschnik, and Andrzej Kaczmarczyk. Multistage committee election. CoRR, abs/2005.02300, 2020. arXiv:2005.02300. 2

10 Jonathan F. Buss and Judy Goldsmith. Nondeterminism within P. SIAM Journal on Computing, 22(3):560-572, 1993. doi:https://doi.org/10.1137/0222038, 10

11 Jiehua Chen, Hendrik Molter, Manuel Sorge, and Ondrej Suchý. Cluster Editing in Multi-Layer and Temporal Graphs. In 29th International Symposium on Algorithms and Computation (ISAAC 2018), volume 123 of LIPIcs, pages 24:1-24:13. Schloss Dagstuhl Leibniz-Zentrum für Informatik, 2018. doi:10.4230/LIPIcs.ISAAC. 2018.24. 9

12 Peter Damaschke. Parameterized enumeration, transversals, and imperfect phylogeny reconstruction. Theor. Comput. Sci., 351(3):337-350, 2006. doi:10.1016/j.tcs. 2005 10.004, 3, 6, 10,23 
13 Rodney G. Downey and Michael R. Fellows. Fundamentals of Parameterized Complexity. Texts in Computer Science. Springer, 2013. doi:10.1007/978-1-4471-5559-1, 11, 19 , 21, 22, 23

14 David Eisenstat, Claire Mathieu, and Nicolas Schabanel. Facility location in evolving metrics. In Proceedings of 41 st International Colloquium on Automata, Languages, and Programming (ICALP 2014), pages 459-470. Springer, 2014. doi:10.1007/ 978-3-662-43951-7_39. 2

15 Stefan Fafianie and Stefan Kratsch. Streaming kernelization. In Proc. 39th International Symposium on Mathematical Foundations of Computer Science 2014 (MFCS 2014), Part II, volume 8635 of LNCS, pages 275-286. Springer, 2014. doi:10.1007/ 978-3-662-44465-8\_24, 10

16 Till Fluschnik, Rolf Niedermeier, Valentin Rohm, and Philipp Zschoche. Multistage vertex cover. In Proceedings of the 14th International Symposium on Parameterized and Exact Computation (IPEC 2019), pages 14:1-14:14. Schloss Dagstuhl - Leibniz-Zentrum für Informatik, 2019. arXiv:1906.00659, doi:10.4230/LIPIcs.IPEC.2019.14, $2,3,9$

17 Till Fluschnik, Rolf Niedermeier, Carsten Schubert, and Philipp Zschoche. Multistage $s$ - $t$ path: Confronting similarity with dissimilarity. CoRR, abs/2002.07569, 2020. arXiv: 2002.07569, 2, 3, 20,

18 Anupam Gupta, Kunal Talwar, and Udi Wieder. Changing bases: Multistage optimization for matroids and matchings. In Proceedings of 41 st International Colloquium on Automata, Languages, and Programming (ICALP 2014), pages 563-575. Springer, 2014. doi: 10.1007/978-3-662-43948-7_47, 2, 3

19 Sepp Hartung and Rolf Niedermeier. Incremental list coloring of graphs, parameterized by conservation. Theor. Comput. Sci., 494:86-98, 2013. doi:10.1016/j.tcs.2012.12.049. 24

20 Pinar Heggernes, Pim van’t Hof, Benjamin Lévêque, Daniel Lokshtanov, and Christophe Paul. Contracting graphs to paths and trees. Algorithmica, 68(1):109-132, 2014. doi: 10.1007/s00453-012-9670-2, 10

21 Petter Holme and Jari Saramäki (eds.). Temporal Networks. Springer, 2013. 3

22 Petter Holme and Jari Saramäki (eds.). Temporal Network Theory. Springer, 2019. 3

23 Frank Kammer and Andrej Sajenko. FPT-space graph kernelizations. CoRR, abs/2007.11643, 2020. arXiv:2007.11643. 10

24 R. Krithika, Abhishek Sahu, and Prafullkumar Tale. Dynamic parameterized problems. Algorithmica, 80(9):2637-2655, 2018. doi:10.1007/s00453-017-0349-6. 2, 24

25 Junjie Luo, Hendrik Molter, André Nichterlein, and Rolf Niedermeier. Parameterized dynamic cluster editing. In Proceedings of the 38th IARCS Annual Conference on Foundations of Software Technology and Theoretical Computer Science, (FSTTCS 2018), pages 46:1-46:15, 2018. Full version to appear in Algorithmica. arXiv:1810.06625 doi:10.4230/LIPICs.FSTTCS.2018.46, 2, 9, 24,

26 George B. Mertzios, Hendrik Molter, Rolf Niedermeier, Viktor Zamaraev, and Philipp Zschoche. Computing maximum matchings in temporal graphs. In Proceedings of the 37th International Symposium on Theoretical Aspects of Computer Science (STACS 2020), 2020. doi:10.4230/LIPICs.STACS.2020.27. 22

27 Valentin Rohm. Vertex cover under time constraints. Bachelor Thesis, TU Berlin, 2018. 2 